\title{
UPLC-ESI-MS/MS Profile and Antioxidant, Cytotoxic, Antidiabetic, and Antiobesity Activities of the Aqueous Extracts of Three Different Hibiscus Species
}

\author{
Hanan M. Al-Yousef ${ }^{D},{ }^{1}$ Wafaa H. B. Hassan, ${ }^{2}$ Sahar Abdelaziz, ${ }^{2}$ Musarat Amina $\left(\mathbb{D},{ }^{1}\right.$ \\ Rasha Adel, ${ }^{2}$ and May A. El-Sayed ${ }^{2}$ \\ ${ }^{1}$ Department of Pharmacognosy, College of Pharmacy, King Saud University, Riyadh, Saudi Arabia \\ ${ }^{2}$ Department of Pharmacognosy, Faculty of Pharmacy, Zagazig University, Zagazig, Egypt \\ Correspondence should be addressed to Hanan M. Al-Yousef; halyousef@ksu.edu.sa
}

Received 8 February 2020; Revised 20 May 2020; Accepted 3 June 2020; Published 25 June 2020

Academic Editor: Casimiro Mantell

Copyright (c) 2020 Hanan M. Al-Yousef et al. This is an open access article distributed under the Creative Commons Attribution License, which permits unrestricted use, distribution, and reproduction in any medium, provided the original work is properly cited.

\begin{abstract}
The aqueous extracts of Hibiscus calyphyllus (HcA), Hibiscus micranthus (HmA), and Hibiscus deflersii (HdA) growing in Saudi Arabia did not receive enough attention in phytochemical and biological studies. This inspired the authors to investigate the phytochemicals of these extracts for the first time using UPLC-ESI-MS/MS in negative and positive ionization modes. The analysis afforded the tentative identification of 103 compounds including phenolic compounds, flavonoids, and anthocyanins. Moreover, in vitro evaluations of their cytotoxic, antioxidant, antidiabetic, and antiobesity activities were carried out. The results showed that aqueous extract of Hibiscus calyphyllus had the highest activity as an antioxidant agent $\left(\mathrm{SC}_{50}=111 \pm 1.5 \mu \mathrm{g} / \mathrm{mL}\right) \mathrm{compared} \mathrm{with}$ ascorbic acid $\left(\mathrm{SC}_{50}=14.2 \pm 0.5 \mu \mathrm{g} / \mathrm{mL}\right)$. MTT assay was used to evaluate cytotoxic activity compared to cisplatin. Hibiscus deflersii showed the most potent cytotoxic effect against A-549 (human lung carcinoma) with $\mathrm{IC}_{50}=50 \pm 5.1 \mu \mathrm{g} / \mathrm{mL}$, and Hibiscus micranthus showed a close effect with $\mathrm{IC}_{50}=60.4 \pm 1.7 \mu \mathrm{g} / \mathrm{mL}$. Hibiscus micranthus showed the most potent effect on HCT-116 (human colon carcinoma) with $\mathrm{IC}_{50}=56 \pm 1.9 \mu \mathrm{g} / \mathrm{mL}$ compared with cisplatin $\left(\mathrm{IC}_{50}=7.53 \pm 3.8 \mu \mathrm{g} / \mathrm{mL}\right.$ ). HcA and $\mathrm{HdA}$ extracts showed weak cytotoxic activity against A-549 and HCT-116 cell lines compared to the other extracts. Eventually, Hibiscus deflersii showed astonishing antidiabetic $\left(\mathrm{IC}_{50}=56 \pm 1.9 \mu \mathrm{g} / \mathrm{mL}\right)$ and antiobesity $\left(\mathrm{IC}_{50}=95.45 \pm 1.9 \mu \mathrm{g} / \mathrm{mL}\right)$ activities using in vitro $\alpha$-amylase inhibitory assay (compared with acarbose $\left(\mathrm{IC}_{50}=34.71 \pm 0.7 \mu \mathrm{g} / \mathrm{mL}\right.$ )) and pancreatic lipase inhibitory assay (compared with orlistat $\left.\left(\mathrm{IC}_{50}=23.8 \pm 0.7 \mu \mathrm{g} / \mathrm{mL}\right)\right)$, respectively. In conclusion, these findings are regarded as the first vision of the phytochemical constituents and biological activities of different Hibiscus aqueous extracts. Hibiscus deflersii aqueous extract might be a hopeful origin of functional constituents with anticancer (on A-549 cell line), antidiabetic, and antiobesity activities. It might be a natural alternative remedy and nutritional policy for diabetes and obesity treatment without negative side effects. Isolation of the bioactive phytochemicals from the aqueous extracts of aerial parts of Hibiscus calyphyllus, Hibiscus micranthus, and Hibiscus deflersii and estimation of their biological effects are recommended in further studies.
\end{abstract}

\section{Introduction}

Hibiscus (Malvaceae) consists of approximately 200 species widely distributed in tropical and subtropical regions of the world [1]. Hibiscus is a genus of herbs, shrubs, and trees [2]. Phytochemical investigation of Hibiscus has been reported to contain many classes of secondary metabolites including anthocyanins, flavonoids, steroids, terpenoids, alkaloids, quinones, and sesquiterpene [2].
Many Hibiscus species are valued as ornamental plants and are cultivated in gardens [2]. Fruits of some species are used as food; a soft drink is provided from flowers of some species (H. sabdariffa L.) and also used in food industry, for example, in cakes, wines, syrups, jellies, puddings, and cold or hot beverages and as a colorant for herbal teas [1]. Since ancient times, Hibiscus has been used in traditional folk medicine for different disorders. 
Various pharmacological effects have also been shown for Hibiscus and its components such as antihypertensive, antiatherosclerotic, antioxidant, antihypercholesterolemic, hypolipidemic, antinociceptive, anti-inflammatory, antipyretic, analgesic, antifungal, antibacterial, antifertility, antidiabetic, anticancer, antimutagenic, chemopreventive, anthelminthic, and anticonvulsant activities [1, 3, 4].

Hibiscus deflersii (HdA), which is native to Ethiopia and grown as ornamental plant worldwide, is $1 \mathrm{~m}$ high erect perennial or annual leafy untidy shrub of bright green narrow dentate leaves surrounding bright crimson-red flowers. Many interesting pharmacological activities of HdA had been reported; its leaves extract is used to treat cardiac disorders and diabetes. However, its flower infusion and extract are used as demulcent and emollient, while its decoction is used for the treatment of bronchial catarrh [5].

Hibiscus micranthus ( $\mathrm{HmA}$ ), which is commonly distributed from south to western part of Saudi Arabia, is a $45 \mathrm{~cm}$ shrub with heavy leaves, white flowers, and short pedicels with very distinctive capsules of pea-size fruits and is distributed vastly in Saudi Arabia, India, tropical Africa, and Ceylon. Its flowers and fruits exhibited antidiabetic and laxative activities when used orally, and when applied topically it is used as antidandruff agent. The plant also showed anti-inflammatory, antipyretic, antitumor, antimicrobial, and antiviral activities. Literature detects a wide range of phytochemicals in $\mathrm{HmA}$ as flavonoids, phenolic acids, fatty alcohols, fatty acids, $\beta$-sitosterol, and alkanes [5].

Hibiscus calyphyllus (HcA) is $1 \mathrm{~m}$ high leafy shrub characterized by bright yellow flower with dark red center surrounded by simple wide serrate leaves. It is commonly found in Jazan, south-western region of Saudi Arabia. The ethyl acetate fraction of this plant showed potent antioxidant activity [5].

Hibiscus aerial parts are considered as food crops consumed as hot or cold beverages (aqueous extract). Numerous scientific papers have been published discussing the chemical contents of different fractions of HcA, HdA, and $\mathrm{HmA}$, which showed their high biological effectiveness, having antioxidant, antidiabetic, antiobesity, and cytotoxic activities. For our knowledge, the previous literature on Hibiscus species suffers from insufficiency of detailed information on the phytoconstituents of aqueous extracts of Saudi HdA, HcA, and HmA and their biological activities. Therefore, the aims of the present research were (i) to perform direct analysis of aqueous extracts, which relies on UPLC coupled with ESI-MS/MS detection, (ii) to detect the antioxidant, antidiabetic, antiobesity, and anticancer activities of aqueous extracts of $\mathrm{HdA}, \mathrm{HcA}$, and $\mathrm{HmA}$; and (iii) to anticipate the components responsible for antioxidant, antidiabetic, antiobesity, and anticancer activities.

\section{Materials and Methods}

2.1. Plant Material. Aerial parts of three different species of genus Hibiscus were collected from As-Sarawat mountains, Jabal As-Sahla', and Aseer province, in Saudi Arabia (2,556 m height above sea level), during March 2009. Taxonomical authentication of plant samples was performed by
Prof. Dr. Mohamed Yousef from the Pharmacognosy Department, College of Pharmacy of King Saud University, and voucher specimens (H. calyphyllus no. HA-234, $H$. deflersii no. HA-567, and H. micranthus no. HA-16240) were kept at the herbarium of the department.

2.2. Preparation of Extracts. Air-dried powder aerial parts of selected plant samples $(600 \mathrm{~g})$ were individually extracted with distilled water at $100^{\circ} \mathrm{C}$ with continuously shaking for 3 hrs. The marc of each plant material was extracted thrice under similar conditions by repeating the above-mentioned procedure. The aqueous extracts were then filtered by centrifugation, and the filtrates were pooled. The obtained filtrates were freed from the solvent by freeze-drying to get dark brown solid masses. The weight of resulted residues was $34.02,26.25$, and $36.41 \mathrm{~g}$ for H. calyphyllus (HcA), H. deflersii ( $\mathrm{HdA})$, and $H$. micranthus (HmA), respectively.

2.3. Chemicals and Reagents. Sigma Aldrich (Hamburg, Germany) provided all the chemical and reagents used throughout the experiments, including HPLC grade methanol ( $\geq 99.9 \%)$, acetonitrile, water for ESI-MS analyses, reagent grade formic acid ( $\geq 95 \%)$, 2,2-diphenyl-1picrylhydrazyl (DPPH) (freshly dissolved in methanol at a concentration of $0.004 \%$ ), ascorbic acid (99\%), Dulbecco's Modified Eagle's Medium (DMEM), L-glutamine, dinitrosalicylic acid (DNS, colour reagent), p-nitrophenyl butyrate $(\mathrm{NPB}, \geq 98 \%)$, and potassium phosphate buffer $(\mathrm{pH}$ 6.0). 3-(4,5-Dimethylthiazol-2-yl)-2,5-diphenyltetrazolium bromide (MTT), cisplatin, DMSO, porcine pancreatic lipase and $\alpha$-amylase enzyme, acarbose ( $\geq 95 \%)$, and orlistat $(\geq 98 \%)$ were used as control. Human lung carcinoma (A549) and human colon carcinoma (HCT-116) cell lines were obtained from the American Type Culture Collection (ATCC, Rockville, MD).

2.4. UPLC-ESI-MS/MS. Ultra-performance liquid chromatography with electrospray ionization quadrupole-linear ion trap-tandem mass spectrometry analysis, performed on ESIMS positive and negative ion acquisition mode, was carried out on a XEVO TQD triple quadruple instrument. Method in a multiple-reaction monitoring (MRM) mode was employed for the quantitative determination of phytochemicals. The crude Hibiscus extracts were analyzed by UPLC, in order to obtain chromatographic profiles of the more polar portions of the extracts, which contain phenolic and flavonoid compounds. The samples were dissolved in HPLC grade methanol, filtered through $0.2 \mu \mathrm{m}$ membrane disc filter, and resulting solution concentrations were in the range of 0.2 to $0.5 \mathrm{mg} / \mathrm{mL}$, depending on each crude extract.

The UPLC system was a mass spectrometer, Waters Corporation, Milford, USA. The reverse-phase separations were performed (ACQUITY UPLC BEH C18 Column, $1.7 \mu \mathrm{m}-2.1 \times 50 \mathrm{~mm} ; 50 \mathrm{~mm} \times 1.2 \mathrm{~mm}$ inner diameter; $1.7 \mu \mathrm{m}$ particle size) at $0.2 \mathrm{~m} / \mathrm{mL}$ flow rate. A previously reported gradient program was applied for the analysis [6]. The mobile phase comprised acidified water containing $0.1 \%$ 
formic acid (A) and acidified methanol containing 0.1\% formic acid (B). The employed elution conditions were as follows: $0-2 \mathrm{~min}$, isocratic elution at $10 \% \mathrm{~B} ; 2-5 \mathrm{~min}$, linear gradient from 10 to $30 \% \mathrm{~B} ; 5-15 \mathrm{~min}$, linear gradient from $30 \%$ to $70 \% \mathrm{~B} ; 15-22 \mathrm{~min}$, linear gradient from $70 \%$ to $90 \%$ $\mathrm{B}$; and $22-25 \mathrm{~min}$, isocratic elution at $90 \% \mathrm{~B}$; finally, washing and reconditioning of column were done. Electrospray ionization (ESI) was performed in both negative and positive ion modes to obtain more data. The parameters for analysis were set using negative ion mode as follows: source temperature $150^{\circ} \mathrm{C}$, cone voltage $30 \mathrm{eV}$, capillary voltage $3 \mathrm{kV}$, desolvation temperature $440^{\circ} \mathrm{C}$, cone gas flow $50 \mathrm{~L} / \mathrm{h}$, and desolvation gas flow $900 \mathrm{~L} / \mathrm{hr}$. Mass spectra were detected in the ESI between $\mathrm{m} / z 100$ and 1000 atomic mass units. Chemical constituents were identified by their ESIQqQLIT-MS/MS spectra and fragmentation patterns. The peaks and spectra were processed using the MassLynx 4.1 software and tentatively identified by comparing their retention time $\left(R_{\mathrm{t}}\right)$ and mass spectrum with reported data and library search (such as FooDB (http://www.Foodb.ca)).

2.5. Antioxidant Activity. The antioxidant activity of extracts was determined at the Regional Center for Mycology and Biotechnology (RCMB) at Al-Azhar University by the DPPH free radical scavenging assay in triplicate, and average values were considered.

2.5.1. DPPH Radical Scavenging Activity [7]. Freshly prepared $(0.1 \mathrm{mM})$ solution of 2,2-diphenyl-1-picrylhydrazyl (DPPH) and different tested extracts prepared at 5, 10, 20, $40,80,160$, and $320 \mu \mathrm{g} / \mathrm{mL}$ in methanol were vigorously mixed and allowed to stand for $30 \mathrm{~min}$ at room temperature in the dark [8]. The absorbance values of the resulting solution were recorded with a UV-visible spectrophotometer (Milton Roy, Spectronic 1201) at $\lambda_{\max }$ of $517 \mathrm{~nm}$ against DPPH radical without antioxidant (control) and the reference compound ascorbic acid $(5,10,20,40,80,160$, and $320 \mu \mathrm{g} / \mathrm{mL}$ ). All the determinations were performed in three replicates and averaged. The percentage inhibition of the DPPH radical was calculated according to the following formula:

$$
\% \mathrm{DPPH} \text { radical }- \text { scavenging }=\left[\left\{\frac{(\mathrm{AC}-\mathrm{AS})}{\mathrm{AC}}\right\} \times 100\right],
$$

where AC is the absorbance of the control solution and AS is the absorbance of the sample in DPPH solution.

The percentage of DPPH radical scavenging was plotted against each extract concentration and ascorbic acid $(\mu \mathrm{g} /$ $\mathrm{mL}$ ) to determine scavenging capacity $\left(\mathrm{SC}_{50}\right)$, which is the concentration required to scavenge $\mathrm{DPPH}$ by $50 \%$ (i.e., concentration giving $50 \%$ reduction in the absorbance of a DPPH solution from its initial absorbance).

2.6. Evaluation of Cytotoxicity. $\mathrm{HdA}, \mathrm{HmA}$, and $\mathrm{HcA}$ were tested for their cytotoxic activity against human lung carcinoma (A-549) and human colon carcinoma (HCT-116) cell lines using 3-(4,5-dimethylthiazol-2-yl)-2,5-diphenyltetrazolium bromide (MTT) against DMSO and cisplatin as negative and positive controls, respectively. These mammalian cell lines were obtained from American Type Culture Collection (ATCC, Rockville, MD). The cells were propagated on Dulbecco's Modified Eagle's Medium (DMEM) supplemented with $10 \%$ heat-inactivated fetal bovine serum, $1 \%$ L-glutamine, HEPES buffer, and $50 \mu \mathrm{g} / \mathrm{mL}$ gentamycin. The cells were maintained at $37^{\circ} \mathrm{C}$ in a humidified atmosphere with $5 \% \mathrm{CO}_{2}$ and were subcultured two to three times a week.

For antitumor assay, the tumor cell lines were suspended in medium at concentration of $5 \times 10^{4}$ cell/well in Corning ${ }^{\circledR}$ 96-well tissue culture plates and then incubated for $24 \mathrm{hrs}$. The tested extracts were then added to 96-well plates (six replicates) to achieve eight concentrations for each extract ranging from $1 \mu \mathrm{g} / \mathrm{mL}$ to $500 \mu \mathrm{g} / \mathrm{mL}$. Six vehicle controls with media or $0.5 \%$ DMSO were run for each 96-well plate as a control. After incubation for $24 \mathrm{~h}$, the numbers of viable cells were determined by the MTT test. Briefly, the media were removed from the 96-well plates and replaced with $100 \mu \mathrm{L}$ of fresh culture DMEM without phenol red; then, $10 \mu \mathrm{L}$ of the $12 \mathrm{mM} \mathrm{MTT}$ stock solution (5 mg of MTT in $1 \mathrm{~mL}$ of phosphate buffered saline (PBS)) was added to each well including the untreated controls. The 96-well plates were then incubated at $37^{\circ} \mathrm{C}$ and $5 \% \mathrm{CO}_{2}$ for $4 \mathrm{hrs}$. An $85 \mu \mathrm{l}$ aliquot of the media was removed from the wells, and $50 \mu \mathrm{L}$ of DMSO was added to each well, mixed thoroughly with the pipette, and incubated at $37^{\circ} \mathrm{C}$ for $10 \mathrm{~min}$. Then, the optical density was measured at $590 \mathrm{~nm}$ with the microplate reader (Sunrise, Tecan, Inc., USA) to determine the number of viable cells, and the percentage of viability was calculated:

$$
\text { cell viability } \%=\left[1-\left(\frac{\mathrm{OD} t}{\mathrm{OD} c}\right)\right] \times 100 \% \text {, }
$$

where OD $t$ is the mean optical density of wells treated with the tested sample and $\mathrm{OD} c$ is the mean optical density of untreated cells.

The relation between surviving cells and each extract concentration $(1-500 \mu \mathrm{g} / \mathrm{mL})$ was plotted to get the survival curve of each tumor cell line after treatment with the tested extract. The $50 \%$ inhibitory concentration $\left(\mathrm{IC}_{50}\right)$, the concentration required to cause toxic effects in $50 \%$ of intact cells, was estimated from graphic plots of the dose-response curve (log extract concentration on $x$-axis vs. percentage viability from untreated cells on $y$-axis) for each concentration through nonlinear regression analysis (dose-response inhibition, $\log$ inhibitor vs. normalized responsevariable slop) using GraphPad Prism 5 software (GraphPad Software, San Diego, California) [9, 10]. All experiments were repeated at least three times. Results are reported as means \pm SD.

\subsection{In Vitro Antidiabetic Assay}

2.7.1. $\alpha$-Amylase Inhibition Method. In a-amylase inhibition method, the enzyme solution was prepared by dissolving $\alpha$-amylase in $20 \mathrm{mM}$ phosphate buffer (6.9) at a 
concentration of $0.5 \mathrm{mg} / \mathrm{mL}$. One $\mathrm{mL}$ of the extract of various concentrations $(7.81-1000 \mu \mathrm{g} / \mathrm{mL})$ and $1 \mathrm{~mL}$ of enzyme solution were mixed together and incubated at $25^{\circ} \mathrm{C}$ for $10 \mathrm{~min}$. After incubation, $1 \mathrm{~mL}$ of starch (0.5\%) solution was added to the mixture and further incubated at $25^{\circ} \mathrm{C}$ for $10 \mathrm{~min}$. The reaction was then stopped by adding $2 \mathrm{~mL}$ of dinitrosalicylic acid (DNS, colour reagent), heating the reaction mixture in a boiling water bath $(5 \mathrm{~min})$. After cooling, the absorbance was measured calorimetrically at $565 \mathrm{~nm}$. The inhibition percentage was calculated using the following formula: $\%$ inhibition $=(1-\mathrm{As} / \mathrm{Ac}) \times 100$, where Ac is the absorbance of control and As is the absorbance of tested extracts. Acarbose was used as a control [11]. The $\mathrm{IC}_{50}$ value was defined as the concentration of $\alpha$-amylase inhibitor needed to inhibit $50 \%$ of its activity under the assay conditions.

Nonlinear regression analysis using GraphPad Prism 5 software (GraphPad Software, San Diego, California) was conducted to calculate $\mathrm{IC}_{50}$ from graphic plots of the doseresponse curve for each applied concentration. Each experiment was performed in triplicate, and all values are represented as means $\pm \mathrm{SD}$.

\subsection{In Vitro Antiobesity Using Pancreatic Lipase Inhibitory} Assay. The lipase inhibition activity of plant extract was determined by the method in [12]. In this assay, the porcine pancreatic lipase activity was measured using $p$-nitrophenyl butyrate (NPB) as a substrate. Lipase solution $(100 \mu \mathrm{g} / \mathrm{mL})$ was prepared in a $0.1 \mathrm{mM}$ potassium phosphate buffer $(\mathrm{pH}$ 6.0). Samples with different concentrations $(7.81-1000 \mu \mathrm{g} /$ $\mathrm{mL}$ ) were preincubated with $100 \mu \mathrm{g} / \mathrm{mL}$ of lipase for $10 \mathrm{~min}$ at $37^{\circ} \mathrm{C}$. The reaction was then started by adding $0.1 \mathrm{~mL} \mathrm{NPB}$ substrate. After incubation at $37^{\circ} \mathrm{C}$ for $15 \mathrm{~min}$, $p$-nitrophenol amount released in the reaction was measured using multiplate reader. Orlistat was used with the same concentrations as a control. The results were expressed as percentage inhibition, which was calculated using the following formula: inhibitory activity $(\%)=(1-\mathrm{As} / \mathrm{Ac}) \times 100$, where As is the absorbance in the presence of test substance and Ac is the absorbance of control. The $\mathrm{IC}_{50}$ value was defined as the concentration of pancreatic lipase inhibitor required to inhibit $50 \%$ of its activity under the assay conditions. Estimation of $\mathrm{IC}_{50}$ was done from dose-response curve graphic plots for each concentration by nonlinear regression analysis using GraphPad Prism 5 software. Each experiment was performed in triplicate, and all values are represented as means \pm SD of triplicates.

\section{Results and Discussion}

3.1. UPLC-ESI-MS/MS. Identification of the chemical composition of the aqueous extract of the $\mathrm{HdA}, \mathrm{HmA}$, and $\mathrm{HcA}$ was carried out by UPLC-ESI-MS/MS in negative and positive ion modes. Totally, 103 secondary metabolites arranged according to retention time $\left(R_{\mathrm{t}}\right)$ were identified depending on their $\mathrm{MS}^{2}$ information given by the precursor ion's mass, their fragments, known fragmentation patterns for the given classes of compounds, and neutral mass loss, as well as comparison with the available literature and searching in an online database [13] as shown in Table 1. Figure 1 shows the base peak chromatograms of the three aqueous extracts.

3.1.1. Phenolic Compounds. Phenolic acid derivatives are mostly glycosides; their fragmentation stage started with the cleavage of the glycosidic linkage to provide the $\mathrm{m} / z$ of the phenolic acid and the corresponding neutral mass loss of sugar molecules $(-162 \mathrm{Da})$, and then neutral mass losses of hydroxyl $(-18 \mathrm{Da})$, methyl $(-15 \mathrm{Da})$, or carboxylic $(-44 \mathrm{Da})$ groups were helpful in identification of the specific phenolic acid. Methyl gallate (72) [17] and its derivative (85) [39] and syringic acid derivative (76) [33] were identified. Compound 33 and its isomer (89) were tentatively identified as 4hydroxybenzoic acid while compound 55 and its isomer (59) were tentatively identified as 3-hydroxybenzoic acid [13].

Tyrosol (4) and its isomers (26, 67, and 94) were characterized by two fragments: $\mathrm{m} / \mathrm{z} 77$, corresponding to the aromatic ring; $m / z$ 93, corresponding to the phenol group, respectively [15]. Tyrosol precursor ion at $\mathrm{m} / z 121$ does not refer to the $[\mathrm{M}+\mathrm{H}]^{+}$ion, but to the $\left[\mathrm{M}+\mathrm{H}-\mathrm{H}_{2} \mathrm{O}\right]^{+}$ according to [15]; this may be due to in-source fragmentation, even under mild ionization conditions.

3.1.2. Flavone $C$-Glycosides. In negative ionization mode, the presence of $[\mathrm{M}-\mathrm{H}-90]^{-}$and $[\mathrm{M}-\mathrm{H}-120]^{-}$confirmed that the compounds are mono-C-hexosylated flavonoids. The sugar on position 8 can be detected by investigation of $\mathrm{MS}^{2}$ spectrum (i.e., the absence of the fragment peak at $\mathrm{m} / \mathrm{z}$ $[\mathrm{M}-\mathrm{H}-18]^{-}$) as in compound 27 , which was identified as orientin (luteolin-8-C-glucoside) [25, 27, 31, 32], and compound 98, which was tentatively identified as kaempferol-8C-glucoside.

The substitution of the two $C$-glucosides in positions 6 and 8 in compound 20 and its isomer (41) can be confirmed by the characteristic fragments at $\mathrm{m} / z 383$ corresponding to $[\mathrm{M}-\mathrm{H}-120-90]^{-}$and $\mathrm{m} / z 353$ corresponding to $[\mathrm{M}-\mathrm{H}-$ $120-120]^{-}$in MS/MS spectrum. The compound was identified as vicenin (apigenin 6,8-di- $C$-glucoside) [26, 27]. Luteolin $C$-hexoside- $C$-pentoside (30) and its isomer (50) with $[\mathrm{M}-\mathrm{H}]^{-}$at $m / z 579$ showed ion fragments at $m / z 489$ $[\mathrm{M}-\mathrm{H}-90]^{-}, m / z 459$ [M - H-120] $^{-}, m / z 429$ [M - H-150] $^{-}$, $\mathrm{m} / z 369[\mathrm{M}-\mathrm{H}-120-90]^{-}$, and $m / z 339[\mathrm{M}-\mathrm{H}-120-120]^{-}$ [25].

Compound 8 and its isomers $(19,36,43)$ exhibited characteristic fragments at $\mathrm{m} / z 443$ corresponding to $[\mathrm{M}-\mathrm{H}-120]^{-}, m / z 431$ corresponding to $[\mathrm{M}-\mathrm{H}-132]^{-}, m / z$ 353 corresponding to $[\mathrm{M}-\mathrm{H}-120-90]^{-}$, and $\mathrm{m} / z 341$ corresponding to $[\mathrm{M}-\mathrm{H}-132-90]^{-}$in $\mathrm{MS}^{2}$ spectrum that confirm the mono-C-hexoside-C-pentoside substitution in positions 6 and 8 . The compound was identified as apigenin $C$-hexoside- $C$-pentoside $[18,25,40]$.

Schaftoside (apigenin-6-C-glucoside-8-C-riboside) (32) and its isomer (51) showed a pseudomolecular ion peak $[\mathrm{M}+\mathrm{H}]^{+}$at $m / z 565$, and the typical fragmentation pathway of C-glycosylated flavonoids resulted in the formation of ions at $m / z 475[\mathrm{M}+\mathrm{H}-90]^{+}$, corresponding to the loss of an 
TABle 1: Metabolites identified in the aqueous extracts of Hibiscus deflersii (HdA), H. micranthus (HmA), and H. calyphyllus (HcA) using UPLC-ESI-MS in negative and positive ionization modes.

\begin{tabular}{|c|c|c|c|c|c|c|c|c|c|}
\hline $\begin{array}{l}\text { Comp. } \\
\text { no. }\end{array}$ & Compound name & $\begin{array}{c}\mathrm{R}_{\mathrm{t}} \\
(\mathrm{min})\end{array}$ & $\begin{array}{c}{[\mathrm{M}-} \\
\mathrm{H}]^{-} \\
(m / z)\end{array}$ & $\begin{array}{c}{[\mathrm{M}+\mathrm{H}]^{+}} \\
\quad(m / z)\end{array}$ & $\mathrm{MS}^{2}$ fragments $(\mathrm{m} / z)$ & HdA & $\mathrm{HcA}$ & $\mathrm{HmA}$ & References \\
\hline 1 & L-ascorbic acid & 0.30 & & 177 & $133,129,127,113,103,101,57$ & $\sqrt{ }$ & $\sqrt{ }$ & $\sqrt{ }$ & 1 \\
\hline 2 & Oleuropein & 0.82 & 539 & & $377,341,307,215,179$ & $\sqrt{ }$ & & $\sqrt{ }$ & 2 \\
\hline 3 & Succinic acid* & 0.86 & & 119 & 101 & $\sqrt{ }$ & & & - \\
\hline 4 & Tyrosol & 0.93 & & 121 & $103,97,93,89,79,77,73,65,45$ & $\sqrt{ }$ & & & 3 \\
\hline 5 & Sucrose & 0.95 & 341 & & $\begin{array}{c}179(\mathrm{M}-\mathrm{H}-162)^{-}, 161.131,119,117 \\
113,103,101,89,87,71,59\end{array}$ & & & $\sqrt{ }$ & 4 \\
\hline 6 & $\begin{array}{l}\text { Hydroxycitric acid } \\
\text { derivative }\end{array}$ & 1.36 & & 593 & 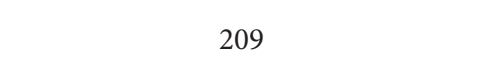 & & $\sqrt{ }$ & & 1 \\
\hline 7 & Butein & 1.43 & & 273 & $163,143,137$ & $\sqrt{ }$ & & & 5 \\
\hline 8 & $\begin{array}{l}\text { Apigenin } C \text {-hexoside- } C \text { - } \\
\text { pentoside }\end{array}$ & 1.53 & 563 & & $\begin{array}{c}545(\mathrm{M}-\mathrm{H}-18)^{-}, 443(\mathrm{M}-\mathrm{H}-120)^{-}, \\
431(\mathrm{M}-\mathrm{H}-132)^{-}, 353(\mathrm{M}-\mathrm{H}-120- \\
90)^{-}, 341(\mathrm{M}-\mathrm{H}-132-90)^{-}, 311 \\
(\mathrm{M}-\mathrm{H}-132-120)^{-}\end{array}$ & & $\sqrt{ }$ & & 6 \\
\hline 9 & L-ascorbic acid isomer & 1.81 & & 177 & $133,129,127,113,103,101,57$ & & & $\sqrt{ }$ & 1 \\
\hline 10 & $N$-feruloyltyramine & 3.32 & & 314 & $235,181,177,145,121,103,93,45$ & & & $\sqrt{ }$ & 1 \\
\hline 11 & Cyanidin 3-O-galactoside & 3.57 & & 449 & 287,137 & & $\sqrt{ }$ & & 7,8 \\
\hline 12 & L-ascorbic acid isomer & 4.52 & & 177 & $133,129,127,113,103,101,57$ & $\sqrt{ }$ & & & 1 \\
\hline 13 & $\begin{array}{c}\text { Patuletin (6- } \\
\text { methoxyquercetin) }\end{array}$ & 4.97 & & 333 & $318,301,169,155$ & $\sqrt{ }$ & & & 9 \\
\hline 14 & Succinic acid isomer & 6.05 & 117 & & $99(\mathrm{M}-\mathrm{H}-18)^{-}$ & $\sqrt{ }$ & & & 10 \\
\hline 15 & $\begin{array}{l}\text { Diosmetin-7-O- } \\
\text { glucuronide- } 3^{\prime}-O- \\
\text { pentoside }\end{array}$ & 6.31 & 607 & & $\begin{array}{c}475(\mathrm{M}-\mathrm{H}-132)^{-}, 299(\mathrm{M}-\mathrm{H}-132- \\
176)^{-}, 179\end{array}$ & & & $\sqrt{ }$ & 11 \\
\hline 16 & L-ascorbic acid isomer & 6.32 & & 177 & $133,129,127,113,103,101,57$ & & $\sqrt{ }$ & & 1 \\
\hline 17 & Succinic acid isomer & 6.49 & 117 & & 117,99 & & & $\sqrt{ }$ & 10 \\
\hline 18 & $\begin{array}{l}\text { Cyanidin 3-O- } \\
\text { sambubioside }\end{array}$ & 6.76 & 579 & & 339,285 & & $\sqrt{ }$ & & 12 \\
\hline 19 & $\begin{array}{l}\text { Apigenin } C \text {-hexoside- } C \text { - } \\
\text { pentoside isomer }\end{array}$ & 7.02 & 563 & & $\begin{array}{c}545(\mathrm{M}-\mathrm{H}-18)^{-}, 443(\mathrm{M}-\mathrm{H}-120)^{-} \\
431(\mathrm{M}-\mathrm{H}-132)^{-}, 341(\mathrm{M}-\mathrm{H}-132- \\
90)^{-}, 311(\mathrm{M}-\mathrm{H}-132-120)^{-}\end{array}$ & $\sqrt{ }$ & & & 13 \\
\hline 20 & $\begin{array}{l}\text { Vicenin (apigenin 6,8-di- } \\
\text { C-glucoside) }\end{array}$ & 7.10 & 593 & & $\begin{array}{c}473(\mathrm{M}-\mathrm{H}-120)^{-}, 383(\mathrm{M}-\mathrm{H}-120- \\
90)^{-}, 285\end{array}$ & $\sqrt{ }$ & & & 14,15 \\
\hline 21 & $\begin{array}{l}\text { Kaempferol-3-O- } \\
\text { rutinoside/luteolin-7-O- } \\
\text { rutinoside }\end{array}$ & 7.25 & 593 & & 285 (M-H-Rut. $)^{-}$ & $\sqrt{ }$ & & & $16 / 17$ \\
\hline 22 & $\begin{array}{l}\text { Apiin (apigenin-7- } \\
\text { apiosylglucoside) }\end{array}$ & 7.29 & 563 & & $443,413,269(\mathrm{M}-\mathrm{H}-132-162)^{-}$ & & $\sqrt{ }$ & & 5,18 \\
\hline 23 & Apiin isomer & 7.31 & & 565 & $\begin{array}{c}433(\mathrm{M}+\mathrm{H}-132)^{+}, 413,271(\mathrm{M}+\mathrm{H}- \\
162-132)^{+}\end{array}$ & $\sqrt{ }$ & & & 5 \\
\hline 24 & Cyanidin 3-O-glucoside & 7.37 & & 494 & $287(\mathrm{M}+\mathrm{H}-162)^{+}, 137$ & $\sqrt{ }$ & & & 3 \\
\hline 25 & $\begin{array}{l}\text { Diosmetin } C \text {-glucoside } \\
\text { C-pentoside* }\end{array}$ & 7.37 & 593 & & $\begin{array}{c}413(\mathrm{M}-\mathrm{H}-90-90)^{-}, 383(\mathrm{M}-\mathrm{H}-90- \\
120)^{-}\end{array}$ & & $\sqrt{ }$ & & - \\
\hline 26 & Tyrosol isomer & 7.46 & & 121 & 93,77 & & $\sqrt{ }$ & & 3 \\
\hline 27 & $\begin{array}{l}\text { Orientin (luteolin } 8-C- \\
\text { glucoside) }\end{array}$ & 7.46 & 447 & & $\begin{array}{l}357(\mathrm{M}-\mathrm{H}-90)^{-}, 327(\mathrm{M}-\mathrm{H}-120)^{-} \\
297(\mathrm{M}-\mathrm{H}-150)^{-}, 285(\mathrm{M}-\mathrm{H}-162)^{-}\end{array}$ & $\sqrt{ }$ & & & $\begin{array}{l}13,15,19, \\
20\end{array}$ \\
\hline 28 & $\begin{array}{l}\text { Delphinidin malonyl } \\
\text { glucuronide* }\end{array}$ & 7.59 & & 565 & $303(\mathrm{M}+\mathrm{H}-176-86)^{+}$ & & $\sqrt{ }$ & & - \\
\hline 29 & $\begin{array}{l}\text { Methyl apigenin } \\
\text { derivative* }^{*}\end{array}$ & 7.81 & & 799 & 285 & $\sqrt{ }$ & & & - \\
\hline 30 & $\begin{array}{l}\text { Luteolin } C \text {-hexoside- } C \text { - } \\
\text { pentoside }\end{array}$ & 7.82 & 579 & & $\begin{array}{c}489\left(\mathrm{M}-\mathrm{H}-90^{-}\right), 459(\mathrm{M}-\mathrm{H}-120)^{-} \\
429(\mathrm{M}-\mathrm{H}-150)^{-}, 411,399,369 \\
(\mathrm{M}-\mathrm{H}-120-90)^{-}, 365,353,339 \\
(\mathrm{M}-\mathrm{H}-120-120)^{-}, 299\end{array}$ & & & $\sqrt{ }$ & 13 \\
\hline 31 & Luteolin derivative & 7.98 & 737 & & 285 & & $\sqrt{ }$ & & 18,21 \\
\hline 32 & $\begin{array}{l}\text { Schaftoside (apigenin 6- } \\
C \text {-glucoside } 8-C- \\
\text { riboside) }\end{array}$ & 7.99 & & 565 & $\begin{array}{c}529,511,475,457,445,427 \\
415(\mathrm{M}+\mathrm{H}-150)^{+}, 409,391,379,361 \\
349,337,325,307,295,273\end{array}$ & & & $\sqrt{ }$ & 1,22 \\
\hline
\end{tabular}


TABle 1: Continued.

\begin{tabular}{|c|c|c|c|c|c|c|c|c|c|}
\hline $\begin{array}{l}\text { Comp. } \\
\text { no. }\end{array}$ & Compound name & $\begin{array}{c}\mathrm{R}_{\mathrm{t}} \\
(\mathrm{min})\end{array}$ & $\begin{array}{l}{[\mathrm{M}-} \\
\mathrm{H}]^{-} \\
(m / z)\end{array}$ & $\begin{array}{l}{[\mathrm{M}+\mathrm{H}]^{+}} \\
\quad(m / z)\end{array}$ & $\mathrm{MS}^{2}$ fragments $(m / z)$ & HdA & $\mathrm{HcA}$ & $\mathrm{HmA}$ & References \\
\hline 33 & 4-Hydroxybenzoic acid & 7.99 & & 139 & $121,111,105,97,93,79$ & & $\sqrt{ }$ & & 1 \\
\hline 34 & $\begin{array}{l}\text { Peonidin-3- }(p- \\
\text { coumaroyl-glucoside })\end{array}$ & 8.27 & & 609 & $301(\mathrm{M}+\mathrm{H}-146-162)^{+}$ & & $\sqrt{ }$ & & 23 \\
\hline 35 & $\begin{array}{l}\text { Kaempferol-3- }(p- \\
\text { coumaryl-glucoside })\end{array}$ & 8.27 & 593 & & $\begin{array}{c}593,447(\mathrm{M}-\mathrm{H}-146)^{-}, 327 \\
285(\mathrm{M}-\mathrm{H}-146-162)^{-}\end{array}$ & & & $\sqrt{ }$ & 24 \\
\hline 36 & $\begin{array}{l}\text { Apigenin } C \text {-hexoside- } C \text { - } \\
\text { pentoside isomer }\end{array}$ & 8.32 & 563 & & $\begin{array}{c}545(\mathrm{M}-\mathrm{H}-18)^{-}, 473(\mathrm{M}-\mathrm{H}-90)^{-} \\
443(\mathrm{M}-\mathrm{H}-120)^{-}, 431(\mathrm{M}-\mathrm{H}- \\
132)^{-}, 353(\mathrm{M}-\mathrm{H}-120-90)^{-}, 341 \\
(\mathrm{M}-\mathrm{H}-132-90)^{-}, 311(\mathrm{M}-\mathrm{H}-132- \\
120)^{-}\end{array}$ & $\sqrt{ }$ & & $\sqrt{ }$ & 13 \\
\hline 37 & $\begin{array}{l}\text { Kaempferol-3-O- } \\
\text { glucoside }\end{array}$ & 8.41 & 447 & & (5) & & $\sqrt{ }$ & & 6,8 \\
\hline 38 & $\begin{array}{l}\text { Gamma-eudesmol } \\
\text { rhamnoside derivative }\end{array}$ & 8.47 & 577 & & $\begin{array}{c}439,397,379,367,349,322,293,249 \\
197,127\end{array}$ & & $\sqrt{ }$ & & 1 \\
\hline 39 & $\begin{array}{l}\text { L-ascorbic acid isomer } \\
\text { Peonidin-3- }(p-\end{array}$ & 8.55 & & 177 & $133,129,127,113,103,101,57$ & $\sqrt{ }$ & & & 1 \\
\hline 40 & $\begin{array}{l}\text { coumaroyl-glucoside) } \\
\text { isomer }\end{array}$ & 8.69 & & 609 & $301(\mathrm{M}+\mathrm{H}-146-162)^{+}$ & $\sqrt{ }$ & $\sqrt{ }$ & & 23 \\
\hline 41 & Vicenin isomer & 8.89 & 593 & & $\begin{array}{c}473(\mathrm{M}-\mathrm{H}-120)^{-}, 395,383(\mathrm{M}-120- \\
90)^{-}, 338,327,298\end{array}$ & & & $\sqrt{ }$ & 15,25 \\
\hline 42 & Apiin isomer & 8.98 & & 565 & $\begin{array}{c}433(\mathrm{M}+\mathrm{H}-132)^{+}, 413,271(\mathrm{M}+\mathrm{H}- \\
162-132)^{+}\end{array}$ & $\sqrt{ }$ & & $\sqrt{ }$ & 5 \\
\hline 43 & $\begin{array}{l}\text { Apigenin } C \text {-hexoside- } C \text { - } \\
\text { pentoside isomer }\end{array}$ & 8.98 & 563 & & $\begin{array}{c}545(\mathrm{M}-\mathrm{H}-18)^{-}, 443(\mathrm{M}-\mathrm{H}-120)^{-} \\
431(\mathrm{M}-\mathrm{H}-132)^{-}, 353(\mathrm{M}-\mathrm{H}-120- \\
90)^{-}, 341(\mathrm{M}-\mathrm{H}-132-90)^{-}, 311 \\
(\mathrm{M}-\mathrm{H}-132-120)^{-}\end{array}$ & $\sqrt{ }$ & & & 6,13 \\
\hline 44 & Unknown & 9.04 & 319 & & $239,204,195,97$ & & & $\sqrt{ }$ & - \\
\hline 45 & $\begin{array}{l}\text { Kaempferol-3- }(p- \\
\text { coumaryl-glucoside }) \\
\text { isomer }\end{array}$ & 9.12 & 593 & & $\begin{array}{c}447(\mathrm{M}-\mathrm{H}-146)^{-}, 327,285(\mathrm{M}-\mathrm{H}- \\
146-162)^{-}\end{array}$ & $\sqrt{ }$ & & & 24 \\
\hline 46 & $\begin{array}{l}\text { Peonidin-3-( } p- \\
\text { coumaroyl-glucoside }) \\
\text { isomer }\end{array}$ & 9.37 & & 609 & $\begin{array}{l}609,579,463(\mathrm{M}+\mathrm{H}-146)^{+}, 301 \\
\quad(\text { peonidin })(\mathrm{M}+\mathrm{H}-146-162)^{+}\end{array}$ & $\sqrt{ }$ & $\sqrt{ }$ & $\sqrt{ }$ & 23 \\
\hline 47 & $\begin{array}{l}\text { Methyl apigenin- } C \text { - } \\
\text { rhamnoside- } O- \\
\text { glucoside* }\end{array}$ & 9.38 & & 593 & $\begin{array}{c}327(\mathrm{M}+\mathrm{H}-162-104)^{+}, 297(\mathrm{M}+\mathrm{H}- \\
162-134)^{+}, 285\end{array}$ & & $\sqrt{ }$ & & - \\
\hline 48 & $\begin{array}{l}\text { Diosmetin-7-O- } \\
\text { rutinoside }\end{array}$ & 9.50 & 607 & & $\begin{array}{c}607,577,461(\mathrm{M}-\mathrm{H}-146)^{-}, 299 \\
(\mathrm{M}-\mathrm{H}-146-162)^{-}\end{array}$ & $\sqrt{ }$ & & $\sqrt{ }$ & 26 \\
\hline 49 & Luteolin hexoside & 9.55 & & 449 & $287(\mathrm{M}+\mathrm{H}-162)^{+}$ & $\sqrt{ }$ & & & 5 \\
\hline 50 & $\begin{array}{l}\text { Luteolin } C \text {-hexoside- } C \text { - } \\
\text { pentoside isomer }\end{array}$ & 9.63 & 579 & & $\begin{array}{c}369(\mathrm{M}-\mathrm{H}-120-90)^{-}, 339(\mathrm{M}-\mathrm{H}- \\
120-120)^{-}, 322,281,259,124 \\
529,511,475,457,445,427\end{array}$ & & $\sqrt{ }$ & & 13 \\
\hline 51 & Schaftoside isomer & 9.94 & & 565 & $\begin{array}{c}415(\mathrm{M}+\mathrm{H}-150)^{+}, 409,391,379,361 \\
349,337,325,307,295,273\end{array}$ & & $\sqrt{ }$ & & 1,22 \\
\hline 52 & Succinic acid isomer & 10.01 & 117 & & 99 & & $\sqrt{ }$ & & 10 \\
\hline 53 & L-ascorbic acid isomer & 10.36 & & 177 & $133,129,127,113,103,101,57$ & & $\sqrt{ }$ & & 1 \\
\hline 54 & Peonidin dirhamnoside* & 10.37 & & 593 & $301(\mathrm{M}+\mathrm{H}-146-146)^{+}$ & $\sqrt{ }$ & & & - \\
\hline 55 & 3-Hydroxybenzoic acid & 10.49 & & 139 & $105,97,93,79$ & & $\sqrt{ }$ & & 1 \\
\hline 56 & $\begin{array}{l}\text { Acacetin- } \\
\text { rhamnoglucoside }\end{array}$ & 10.61 & 591 & & $283(\mathrm{M}-\mathrm{H}-\text { Rut. })^{-}, 103,58$ & $\sqrt{ }$ & & $\sqrt{ }$ & 18 \\
\hline 57 & $\begin{array}{l}\text { Kaempferol dimethyl } \\
\text { ether dipentoside* }\end{array}$ & 10.68 & & 579 & $579,315(\mathrm{M}+\mathrm{H}-132-132)^{+}$ & & & $\sqrt{ }$ & - \\
\hline 58 & $\begin{array}{l}\text { Peonidin glucoside } \\
\text { feruloyl glucuronide }\end{array}$ & 10.82 & & 815 & $\begin{array}{c}463(\mathrm{M}+\mathrm{H}-352 \text { feruloyl } \\
\text { glucuronide })^{+}\end{array}$ & $\sqrt{ }$ & & & 7 \\
\hline 59 & $\begin{array}{l}\text { 3-Hydroxybenzoic acid } \\
\text { isomer }\end{array}$ & 10.94 & & 139 & $105,97,93,79$ & & $\sqrt{ }$ & & 1 \\
\hline 60 & $\begin{array}{c}\mathrm{N} \text {-feruloyltyramine } \\
\text { isomer }\end{array}$ & 10.96 & & 314 & $235,218,181,177,145,121,103,93$ & & & $\sqrt{ }$ & 1 \\
\hline 61 & Unknown & 11.07 & 877 & & $877,813,783,557$ & & $\sqrt{ }$ & & - \\
\hline 62 & Apigenin-O-dihexoside & 11.38 & 593 & & $269(\mathrm{M}-\mathrm{H}-162-162)^{-}$ & & $\sqrt{ }$ & & 9 \\
\hline
\end{tabular}


TABLE 1: Continued.

\begin{tabular}{|c|c|c|c|c|c|c|c|c|c|}
\hline $\begin{array}{l}\text { Comp. } \\
\text { no. }\end{array}$ & Compound name & $\begin{array}{c}\mathrm{R}_{\mathrm{t}} \\
(\min )\end{array}$ & $\begin{array}{c}{[\mathrm{M}-} \\
\mathrm{H}]^{-} \\
(m / z)\end{array}$ & $\begin{array}{c}{[\mathrm{M}+\mathrm{H}]^{+}} \\
\quad(m / z)\end{array}$ & $\mathrm{MS}^{2}$ fragments $(\mathrm{m} / z)$ & $\mathrm{HdA}$ & $\mathrm{HcA}$ & $\mathrm{HmA}$ & References \\
\hline 63 & $\begin{array}{l}\text { Isorhamnetin-3-O- } \\
\text { rutinoside }\end{array}$ & 11.59 & 623 & & 315 (M - H-Rut.) ${ }^{-}, 300,271$ & $\sqrt{ }$ & & & 27 \\
\hline 64 & $\begin{array}{l}\text { Peonidin dirhamnoside } \\
\text { isomer* }\end{array}$ & 11.69 & & 593 & $\begin{array}{c}447(\mathrm{M}+\mathrm{H}-146)^{+}, 301(\mathrm{M}+\mathrm{H}-146- \\
146)^{+}\end{array}$ & $\sqrt{ }$ & $\sqrt{ }$ & & - \\
\hline 65 & $\begin{array}{l}\text { Acacetin- } \\
\text { rhamnoglucoside isomer }\end{array}$ & 11.69 & 591 & & 283 (M - H-Rut. $)^{-}$ & $\sqrt{ }$ & & $\sqrt{ }$ & 18 \\
\hline 66 & Succinic acid isomer & 11.78 & 117 & & 99 & & $\sqrt{ }$ & & 10 \\
\hline 67 & Tyrosol isomer & 12.05 & & 121 & 93,77 & & $\sqrt{ }$ & & 3 \\
\hline 68 & $\begin{array}{l}\text { Diosmetin rhamnoside } \\
\text { feruloyl glucuronide* }\end{array}$ & 12.46 & 797 & & $\begin{array}{c}445(\mathrm{M}-\mathrm{H}-176-176)^{-}, 299(\mathrm{M}-\mathrm{H}- \\
176-176-146)^{-}, 237,205\end{array}$ & $\sqrt{ }$ & & & - \\
\hline 69 & Apiin isomer & 12.87 & 563 & & 269 & $\sqrt{ }$ & & & 18 \\
\hline 70 & Succinic acid isomer & 12.90 & 117 & & 99 & & $\sqrt{ }$ & & 10 \\
\hline 71 & Peonidin dipentoside* & 13.13 & & 565 & $\begin{array}{c}565,301(\mathrm{M}+\mathrm{H}-132-132)^{+}, 336265 \\
195,135,91,45\end{array}$ & $\sqrt{ }$ & & & - \\
\hline 72 & Methyl gallate & 13.25 & & 185 & 168,124 & & $\sqrt{ }$ & & 5 \\
\hline 73 & Succinic acid isomer & 13.65 & 117 & & 99 & $\sqrt{ }$ & & & 10 \\
\hline 74 & $\begin{array}{l}\text { Delphinidin-3- } \\
\text { arabinoside derivative }\end{array}$ & 15.40 & & 799 & $435,303(\mathrm{M}+\mathrm{H}-364-132)^{+}$ & $\sqrt{ }$ & & & 7,23 \\
\hline 75 & $\begin{array}{l}\text { Hydroxy- } \\
\text { octadecadienoic acid } \\
\text { derivative }\end{array}$ & 15.57 & 593 & & $\begin{array}{c}295(\mathrm{M}-\mathrm{H}-298)^{-}, 277(\mathrm{M}-\mathrm{H}-298- \\
18)^{-}, 251\left(\mathrm{M}-\mathrm{H}-298-18-\mathrm{CO}_{2}\right)^{-}, 195 \\
171\end{array}$ & $\sqrt{ }$ & & & 28 \\
\hline 76 & Syringic acid derivative & 15.83 & 377 & & 197 & & $\sqrt{ }$ & & 21 \\
\hline 77 & 22-Dehydrocholesterol & 16.00 & & 393 & $393,273,173,171,130,125$ & & & $\sqrt{ }$ & 1 \\
\hline 78 & Malvidin derivative* & 16.72 & & 565 & 331,147 & $\sqrt{ }$ & & & - \\
\hline 79 & $\begin{array}{l}\text { Kaempferide derivative } \\
\text { isomer }\end{array}$ & 17.40 & 623 & & $299\left(\mathrm{M}-\mathrm{H}-2 \mathrm{Glc}^{-}\right)^{-}, 163$ & $\sqrt{ }$ & & & 29 \\
\hline 80 & Unknown & 17.42 & & 274 & $\begin{array}{c}274,256(\mathrm{M}+\mathrm{H}-18)^{+}, 210(\mathrm{M}+\mathrm{H}-18 \\
-46)^{+}, 111,105,102,88,71\end{array}$ & & $\sqrt{ }$ & $\sqrt{ }$ & - \\
\hline 81 & Unknown & 17.54 & & 399 & $267,253,227$ & $\sqrt{ }$ & & & - \\
\hline 82 & L-ascorbic acid isomer & 17.82 & & 177 & $133,129,127,113,103,101,57$ & & $\sqrt{ }$ & & 1 \\
\hline 83 & Succinic acid isomer & 21.49 & 117 & & 117,99 & & $\sqrt{ }$ & & 10 \\
\hline 84 & Apiin isomer & 21.89 & & 565 & $\begin{array}{c}433(\mathrm{M}+\mathrm{H}-132)^{+}, 413,271(\mathrm{M}+\mathrm{H}- \\
162-132)^{+}\end{array}$ & & & $\sqrt{ }$ & 5 \\
\hline 85 & Methyl gallate derivative & 23.06 & 325 & & $183(\mathrm{M}-\mathrm{H}-142)^{-}$ & $\sqrt{ }$ & & & 29 \\
\hline 86 & Gallocatechin derivative & 24.48 & 561 & & 305 & $\sqrt{ }$ & & & 21 \\
\hline 87 & Succinic acid isomer & 24.96 & 117 & & 99 & & $\sqrt{ }$ & & 10 \\
\hline 88 & Unknown & 25.01 & & 515 & $515,353.331,313,239$ & & & $\sqrt{ }$ & - \\
\hline 89 & $\begin{array}{l}\text { 4-Hydroxybenzoic acid } \\
\text { isomer }\end{array}$ & 25.28 & & 139 & $121,111,105,97,93,79$ & $\sqrt{ }$ & $\sqrt{ }$ & & 1 \\
\hline 90 & Succinic acid isomer & 26.11 & 117 & & $117,101,99$ & $\sqrt{ }$ & $\sqrt{ }$ & & 10 \\
\hline 91 & Unknown & 26.66 & & 805 & $\begin{array}{c}615,606,598,413,391,279,167,149 \\
113\end{array}$ & $\sqrt{ }$ & & & - \\
\hline 92 & $\begin{array}{l}\text { Luteolin-7-glucuronide-3 } \\
\text { '/4' -pentoside }\end{array}$ & 26.77 & 593 & & $\begin{array}{c}461(\mathrm{M}-\mathrm{H}-132)^{-}, 285(\mathrm{M}-\mathrm{H}-132- \\
176)^{-}, 169\end{array}$ & & & $\sqrt{ }$ & 11 \\
\hline 93 & $\begin{array}{l}\text { Peonidin dipentoside } \\
\text { isomer* }\end{array}$ & 27.45 & & 565 & $\begin{array}{c}547,301(\mathrm{M}+\mathrm{H}-132-132)^{+}, 259,219 \\
133,113,85,45\end{array}$ & $\sqrt{ }$ & & $\sqrt{ }$ & - \\
\hline 94 & Tyrosol isomer & 27.55 & & 121 & 93,77 & & $\sqrt{ }$ & & 3 \\
\hline 95 & $\begin{array}{l}\text { L-ascorbic acid isomer } \\
\text { Delphinidin-3- }(p-\end{array}$ & 27.61 & & 177 & $133,129,127,113,103,101,57$ & & & $\sqrt{ }$ & 1 \\
\hline 96 & $\begin{array}{c}\text { coumaroyl-glucoside) } \\
\text { derivative }\end{array}$ & 28.36 & & 799 & $611,303(\mathrm{M}+\mathrm{H}-188-308)^{+}$ & & $\sqrt{ }$ & & 23 \\
\hline 97 & Epicatechin derivative & 28.91 & & 678 & 289 & & $\sqrt{ }$ & & 6 \\
\hline 98 & $\begin{array}{l}\text { Kaempferol-8C- } \\
\text { glucoside }{ }^{*}\end{array}$ & 29.40 & & 449 & $329(\mathrm{M}+\mathrm{H}-120)^{+}, 299(\mathrm{M}+\mathrm{H}-150)^{+}$ & & $\sqrt{ }$ & & - \\
\hline 99 & $\begin{array}{l}\text { Malvidin 3-O-glucoside } \\
\text { derivative }\end{array}$ & 29.83 & & 871 & 493,331 & $\sqrt{ }$ & & & 8 \\
\hline 100 & Unknown & 30.00 & & 871 & $593,552,369,260,105$ & & & $\sqrt{ }$ & - \\
\hline 101 & L-ascorbic acid isomer & 30.41 & & 177 & $133,129,127,113,103,101,57$ & $\sqrt{ }$ & $\sqrt{ }$ & & 1 \\
\hline 102 & Peonidin derivative* & 30.65 & & 799 & $648(\mathrm{M}+\mathrm{H} \text {-galloyl })^{+}, 301$ (peonidin) & & $\sqrt{ }$ & & - \\
\hline 103 & Succinic acid isomer & 31.52 & 117 & & 117,99 & $\sqrt{ }$ & & & 10 \\
\hline
\end{tabular}




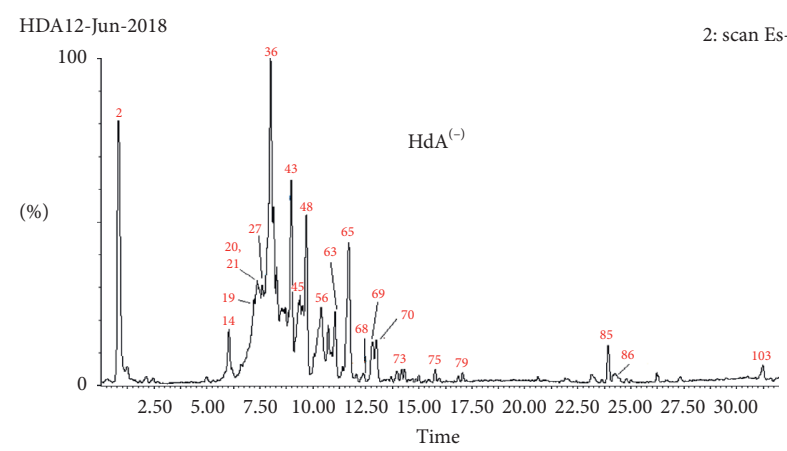

(a)

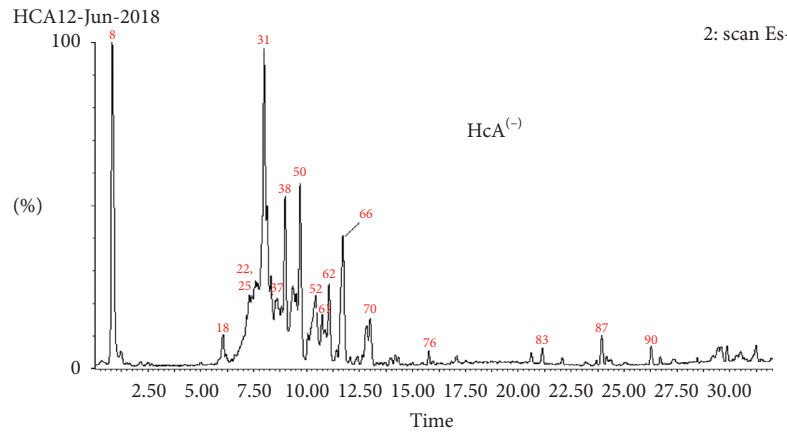

(c)

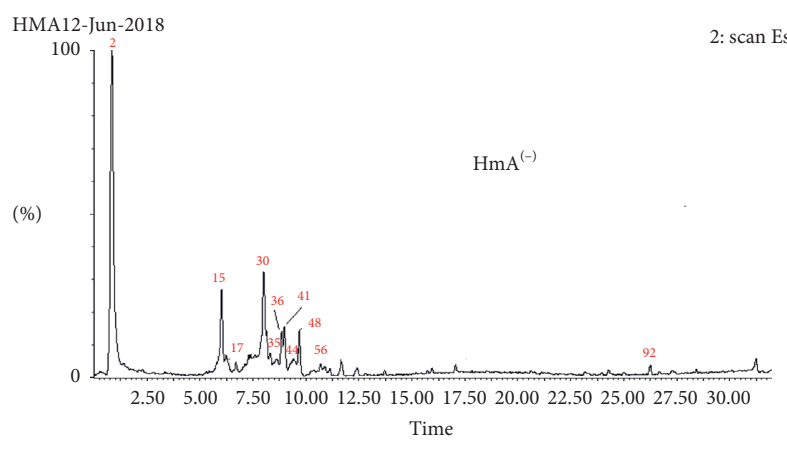

(e)

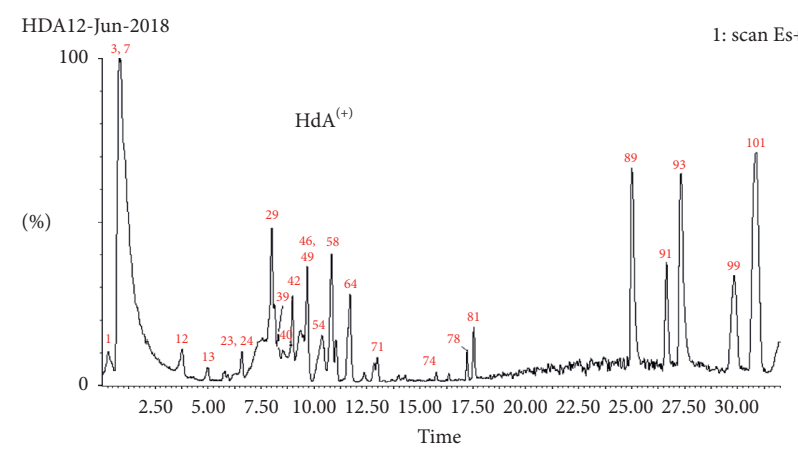

(b)

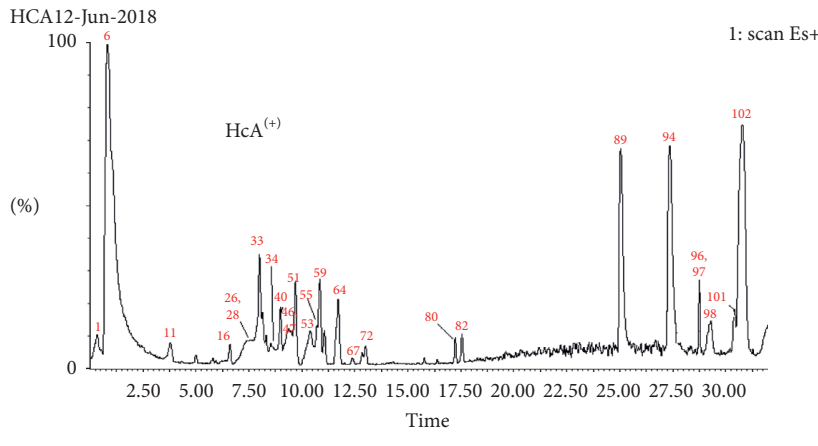

(d)

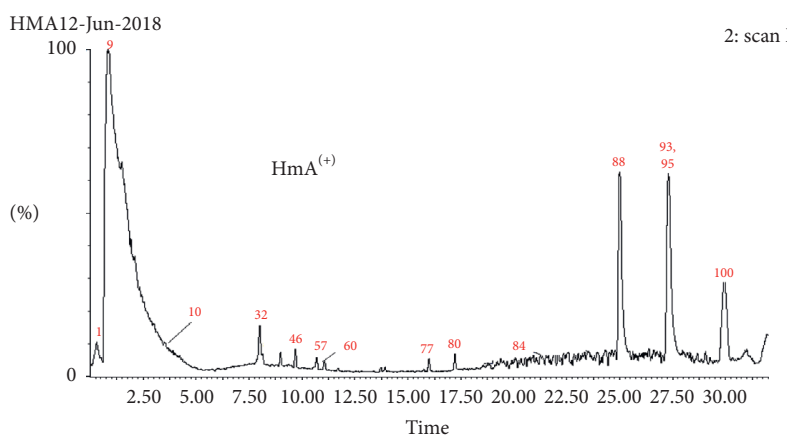

(f)

FIGURE 1: UPLS-ESI-MS chromatograms of aqueous extracts of aerial parts of $H$. deflersii (HdA), H. calyphyllus (HcA), and H. micranthus $(\mathrm{HmA})$ in negative $(-)$ and positive $(+)$ ionization modes.

arabinose unit (riboside), and at $m / z 445[\mathrm{M}+\mathrm{H}-120]^{+}$, corresponding to the loss of a glucose unit. Further fragmentations of the sugar moieties were observed to generate $[\mathrm{M}+\mathrm{H}-186]^{+}$ions at $\mathrm{m} / z 379,[\mathrm{M}+\mathrm{H}-240]^{+}$ions (forming the base peaks) at $m / z 325$, and $[\mathrm{M}+\mathrm{H}-270]^{+}$ions at $m / z 295$ [34].

Diosmetin C-glucoside $C$-pentoside (25) showed a pseudomolecular ion peak $[\mathrm{M}-\mathrm{H}]^{-}$at $m / z$ 593. The typical fragmentation pathway of $C$-glycosylated flavonoids resulted in the formation of ions at $\mathrm{m} / z 413$ [M-H-90-90] $]^{-}$, corresponding to the loss of an pentoside moiety, and at $\mathrm{m} / z 383$ $[\mathrm{M}-\mathrm{H}-120-90]^{-}$, corresponding to the loss of a glucose moiety.

3.1.3. Flavonoid $O, C$-Glycosides. Flavonoid $O, C$-glycosides are distinguished by the lack of the aglycone ion. Only the precursor ion $[\mathrm{M}-\mathrm{H}]^{-}$is detected in addition to the ions resulting from the interglycosidic linkage cleavage including the key fragmentation ions at $[\mathrm{M}-\mathrm{H}-120]^{-}$and $[\mathrm{M}-\mathrm{H}-$ $120-162]^{-}$or $[\mathrm{M}-\mathrm{H}-90-146]^{-}$with or without $[\mathrm{M}-\mathrm{H}-18]^{-}$. On the basis of these rules, compound $47\left(R_{\mathrm{t}} 9.38 \mathrm{~min}\right)$ with a $[\mathrm{M}+\mathrm{H}]^{+}$ion at $\mathrm{m} / \mathrm{z} 593$ was tentatively identified as methyl apigenin- $C$-rhamnoside- $O$-glucoside as it showed $\mathrm{MS}^{2}$ fragments at $m / z 327[\mathrm{M}+\mathrm{H}-162-104]^{+}$, corresponding to loss of $\mathrm{O}$-glucoside moiety $(-162 \mathrm{Da}), \mathrm{m} / z 297[\mathrm{M}+\mathrm{H}-$ $162-134]^{+}$, corresponding to additional loss of $C$-rhamnosyl moiety (-134 Da), and $\mathrm{m} / z 285$ (methyl apigenin).

3.1.4. Flavonoid O-Glycosides. Fragmentation pattern of flavonoid $O$-glycosides is characterized by the loss of the sugar moiety [41, 42], and as a result a deprotonated aglycone ion is yielded in $\mathrm{MS}^{2}$. Compound $21\left(R_{\mathrm{t}} 7.25 \mathrm{~min}\right)$ with $[\mathrm{M}-\mathrm{H}]^{-}$ion peak at $\mathrm{m} / z 593$ was tentatively identified as kaempferol-3-O-rutinoside/luteolin-7-O-rutinoside 
based on the ESI mass data and the $308 \mathrm{Da}$ neutral loss leaving fragment ion at $\mathrm{m} / \mathrm{z} 285$ corresponding to kaempferol/luteolin aglycones $[29,37]$. Diosmetin-7-O-glucuronide-3'-O-pentoside (15) $\left(R_{\mathrm{t}} 6.31 \mathrm{~min}\right)$ produced a $[\mathrm{M}-\mathrm{H}]^{-}$ ion at $m / z 607$ with $\mathrm{MS}^{2}$ fragments at $m / z 475[\mathrm{M}-\mathrm{H}-132]^{-}$, corresponding to loss of pentosyl moiety, and $299[\mathrm{M}-\mathrm{H}-$ $132-176]^{-}$, corresponding to loss of additional glucuronyl moiety [23].

Apigenin-7-O-apiosylglucoside (apiin) (22) and its isomer (69) were tentatively identified from the MS profile of peak at $R_{\mathrm{t}}$ of 7.29 and $12.87 \mathrm{~min}$ with $[\mathrm{M}-\mathrm{H}]^{-}$at $\mathrm{m} / z 563$ and MS/MS base peak fragment ion at $m / z 269$, which gave the loss of $294 \mathrm{Da}(162+132 \mathrm{Da})$ (apiosylglucoside moiety) $[17,30]$. Furthermore, other three apiin isomers $(23,42$, and 84) were tentatively identified from the MS profile of peaks at retention time of $7.31,8.98$, and $21.89 \mathrm{~min}$ with $[\mathrm{M}+\mathrm{H}]^{+}$ at $\mathrm{m} / z 565$ and $\mathrm{MS}^{2}$ base peak fragment ion at $\mathrm{m} / z 433$ $[\mathrm{M}+\mathrm{H}-132]^{+}$, corresponding to apiosyl moiety loss, and at $\mathrm{m} / \mathrm{z} 271$, which indicated the loss of $294 \mathrm{Da}(162+132 \mathrm{Da})$ (apiosylglucoside moiety) [17]. Compounds 35 and 45 with $[\mathrm{M}-\mathrm{H}]^{-}$ion at $m / z 593$ were tentatively identified as kaempferol-3-O-( $p$-coumaryl glucoside). The fragmentation pattern showed $\mathrm{MS}^{2}$ fragments at $\mathrm{m} / z 447[\mathrm{M}-\mathrm{H}-146]^{-}$, corresponding to loss of rhamnose $(-146 \mathrm{Da})$ moiety, and at $\mathrm{m} / z 285$ [M - H-146-162] ${ }^{-}$, corresponding to additional loss of glucose moiety $(-162 \mathrm{Da})$. They both gave the product ion at $m / z 285$ corresponding to the kaempferol aglycone [28]. Compound $37\left(R_{\mathrm{t}} 8.41 \mathrm{~min}\right)$ with $[\mathrm{M}-\mathrm{H}]^{-}$at $\mathrm{m} / z 447$ was tentatively identified as kaempferol-3-O-glucoside from ESI $^{-}$mass data and the neutral loss of $162 \mathrm{Da}$ for glucosyl moiety $[18,20]$. By the same manner, compound $49\left(R_{\mathrm{t}}\right.$ $9.55 \mathrm{~min})$ was identified as luteolin hexoside $\left([\mathrm{M}+\mathrm{H}]^{+}\right.$ion at $\mathrm{m} / z 449$ and $\mathrm{MS}^{2}$ ion at $\left.\mathrm{m} / z 287(\mathrm{M}+\mathrm{H} \text {-glc. }]^{+}\right)$.The precursor ion of compound 48 was detected at $\mathrm{m} / z 607$ $[\mathrm{M}-\mathrm{H}]^{-}$and its characteristic $\mathrm{MS}^{2}$ fragment ion at $\mathrm{m} / z 299$ [M-H-rut. $]^{-}$, related to deprotonated diosmetin, and consequently it was tentatively identified as diosmetin-7-Orutinoside [36]. Additionally the precursor ion of compounds 56 and 65 was detected at $m / z 591[\mathrm{M}-\mathrm{H}]^{-}$and its characteristic $\mathrm{MS}^{2}$ fragment ion at $m / z 283$ [M-H-rut.] ${ }^{-}$, related to deprotonated acacetin, and consequently it was tentatively identified as acacetin-rhamnoglucoside and its positional isomers [30]. Compound 57 was tentatively identified as kaempferol dimethyl ether dipentoside $\left(R_{\mathrm{t}}\right.$ $10.68 \mathrm{~min})$ as it produced a $[\mathrm{M}+\mathrm{H}]^{+}$ion at $\mathrm{m} / z 579$ with $\mathrm{MS}^{2}$ fragments at $m / z 315[\mathrm{M}+\mathrm{H}-132-132]^{+}$, corresponding to loss of dipentosyl moieties. Compound 62 with a $[\mathrm{M}-\mathrm{H}]-$ at $m / z 593$ and $\mathrm{MS}^{2}$ ions at $m / z 269$ [M $-\mathrm{H}-162$ $162]^{-}$was tentatively identified as apigenin-O-dihexoside [21]. Compound $63[\mathrm{M}-\mathrm{H}]^{-}$ion at $\mathrm{m} / z 623$ was tentatively identified as isorhamnetin-3-O-rutinoside. The fragmentation patterns showed $\mathrm{MS}^{2}$ fragments at $m / z 315[\mathrm{M}-\mathrm{H}$ $308]^{-}$(isorhamnetin), corresponding to loss of rutinose $(-308 \mathrm{Da})$ moiety [37]. Compound 68 with $[\mathrm{M}-\mathrm{H}]^{-}$at $\mathrm{m} / \mathrm{z}$ 797 was tentatively identified as diosmetin rhamnoside feruloyl glucuronide as it showed $\mathrm{MS}^{2}$ fragments at $\mathrm{m} / \mathrm{z} 445$ $[\mathrm{M}-\mathrm{H}-176-176]^{-}$, corresponding to loss of feruloyl $(-176 \mathrm{Da})$ and glucuronide $(-176 \mathrm{Da})$ moieties, and 299 $[\mathrm{M}-\mathrm{H}-176-176-146]^{-}$, corresponding to additional loss of rhamnose moiety (-146 Da). According to [39], kaempferide derivative (79) was tentatively identified by its molecular ion $[\mathrm{M}-\mathrm{H}]^{-}$at $m / z 623$ and fragmentation pattern containing specific ion at $\mathrm{m} / z 299[\mathrm{M}-\mathrm{H}-162-162]^{-}$, corresponding to loss of two glucose moieties, and ion at $\mathrm{m} / z$ 163. According to [23], the diglycosylated flavonoid (92) was detected as it possessed a pseudomolecular ion $[\mathrm{M}-\mathrm{H}]^{-}$at $m / z 593$ with $\mathrm{MS}^{2}$ fragment ions at $\mathrm{m} / z 461[\mathrm{M}-\mathrm{H}-132]^{-}$, corresponding to pentosyl moiety loss, as well as other less abundant ion at $\mathrm{m} / \mathrm{z} 285$ [M - H- 132-176] $]^{-}$resulting from the loss of glucuronyl moiety $(-176 \mathrm{Da})$. This compound was identified as luteolin-7-glucuronide-3'/4'-pentoside.

3.1.5. Flavonoid Aglycones. Patuletin (6-methoxy quercetin) (13) was identified by comparing its $\mathrm{MS}^{2}$ fragmentation pattern with the previously reported data [21]. Compound 31 with $[\mathrm{M}-\mathrm{H}]^{-}$ion at $m / z 737$ and $\mathrm{MS}^{2}$ fragment ion at $\mathrm{m} / \mathrm{z}$ 285 was determined to be luteolin derivative $[30,33]$, while compound 29 , which gave a $[\mathrm{M}+\mathrm{H}]^{+}$ion at $\mathrm{m} / z 799$ and $\mathrm{MS}^{2}$ fragment ion at $\mathrm{m} / z 285$, was identified tentatively as methyl apigenin derivative. Gallocatechin derivative (86) [33] and epicatechin derivative (97) [18] were recognized by comparing their $\mathrm{MS}^{2}$ fragmentation pattern with the previously published data.

3.1.6. Anthocyanins. A total of 9 anthocyanin derivatives have been detected in Hibiscus. Compounds 34, 40, 46, 54, $58,64,71,93$, and 102 were tentatively identified as peonidin derivatives. Thus, mass data of compound 34 and its isomers (40 and 46) were proposed to be peonidin-3-( $p$-coumaroylglucoside) as they showed a molecular ion peak $[\mathrm{M}+\mathrm{H}]^{+}$at $\mathrm{m} / \mathrm{z} 609$ and $\mathrm{MS}^{2}$ fragment ions at $\mathrm{m} / z$ 301 $[\mathrm{M}+\mathrm{H}-162-$ $146]^{+}$. These were indicative of a peonidin with glucose (162 Da) and coumaroyl (146 Da) moieties [35].

Compounds 71 and 93 produced a $[\mathrm{M}+\mathrm{H}]^{+}$ion at $\mathrm{m} / \mathrm{z}$ 565 with $\mathrm{MS}^{2}$ fragments at $m / z 301[\mathrm{M}+\mathrm{H}-132-132]^{+}$. These were indicative of a peonidin with two pentoside moieties $(264 \mathrm{Da})$ and were tentatively identified as peonidin dipentoside, while compounds 54 and 64 produced $[\mathrm{M}+\mathrm{H}]^{+}$ ion at $m / z 593$ with $\mathrm{MS}^{2}$ fragments at $m / z 301[\mathrm{M}+\mathrm{H}-146$ $146]^{+}$(peonidin). These were indicative of peonidin with two rhamnoside moieties $(292 \mathrm{Da})$ and were tentatively identified as peonidin dirhamnoside. Compound 58 was tentatively identified as peonidin glucoside feruloyl glucuronide as it produced a $[\mathrm{M}+\mathrm{H}]^{+}$ion at $m / z 815$ with $\mathrm{MS}^{2}$ fragment ions at $m / z 463$ [M $+\mathrm{H}-176-176]^{+}$, corresponding to the loss of a feruloyl $(-176 \mathrm{Da})$ and glucuronide $(-176 \mathrm{Da})$ moieties, and at $m / z 301[\mathrm{M}+\mathrm{H}-176-176-162]^{+}$(peonidin), corresponding to the loss of a glucose unit. Compound 102 was tentatively identified as peonidin derivative as it showed a $[\mathrm{M}+\mathrm{H}]^{+}$ion at $m / z 799$ with $\mathrm{MS}^{2}$ fragment ions at $m / z 648$ $[\mathrm{M}+\mathrm{H}-151]^{+}$, corresponding to the loss of a galloyl $(-151 \mathrm{Da})$ moiety, and at $\mathrm{m} / \mathrm{z} 301$ (peonidin).

The mass spectra of compounds 11 and 24 showed their protonated aglycon ions $[\mathrm{M}+\mathrm{H}]^{+}$to be $m / z 287$, corresponding to cyanidin. These protonated aglycon ions were all formed by loss of a sugar moiety with 162 units from their $[\mathrm{M}+\mathrm{H}]^{+}$, indicating that they are anthocyanidin 
monoglucosides or monogalactosides. This suggests the presence of cyanidin 3-O-galactoside (11) and cyanidin 3-Oglucoside (24). The $m / z$ values of compound 18 detected at $\mathrm{m} / z 579$ in the negative ion mode are similar to those of cyanidin 3-O-sambubioside [24].

Compounds 28, 74, and 96 were tentatively identified as delphinidin derivatives. Compound 28 showed a molecular ion peak $[\mathrm{M}+\mathrm{H}]^{+}$at $m / z 565$ and $\mathrm{MS}^{2}$ fragment ions at $\mathrm{m} / z$ $303[\mathrm{M}+\mathrm{H}-176-86]^{+}$. These were indicative of a delphinidin with glucuronide $(176 \mathrm{Da})$ and malonyl $(86 \mathrm{Da})$ moieties. This compound was suggested to be delphinidin malonyl glucuronide.

Compound 74 showed a molecular ion peak $[\mathrm{M}+\mathrm{H}]^{+}$at $m / z 799$ and $\mathrm{MS}^{2}$ fragment ions at $m / z 435[\mathrm{M}+\mathrm{H}-364]^{+}$ (delphinidin-3-arabinoside) and $m / z 303$ [M $+\mathrm{H}-364-132]^{+}$, corresponding to loss of arabinose moiety. These were indicative of a delphinidin-3-arabinoside derivative [19, 35]. Compound 96 showed a molecular ion peak $[\mathrm{M}+\mathrm{H}]^{+}$at $\mathrm{m} / z$ 799 and $\mathrm{MS}^{2}$ fragment ions at $m / z 611[\mathrm{M}+\mathrm{H}-188]^{+}$and at $\mathrm{m} / z 303[\mathrm{M}+\mathrm{H}-188-308]^{+}$. These were indicative of a delphinidin with coumaroyl-glucoside moieties (-308 Da) [43]. This compound was proposed to be delphinidin-3-( $p$-coumaroyl-glucoside) derivative [35].

Compound 78 was tentatively identified as malvidin derivative as it showed a protonated aglycone ion peak at $\mathrm{m} /$ $z 331$ corresponding to malvidin [43]. Compound 99 produced a $[\mathrm{M}+\mathrm{H}]^{+}$ion at $m / z 871$ with $\mathrm{MS}^{2}$ fragments at $m / z$ 493, corresponding to malvidin-3-O-glucoside [20], and at $\mathrm{m} / z 331[\mathrm{M}+\mathrm{H}-378-162]^{+}$, corresponding to malvidin [43], with one glucose moiety (162 Da), and was identified as malvidin 3-O-glucoside derivative [20].

3.1.7. Fatty Acid Derivatives. For compound 75, a pseudomolecular ion peak $[\mathrm{M}-\mathrm{H}]^{-}$at $\mathrm{m} / z 593$ was observed with $\mathrm{MS}^{2}$ fragment ions at $m / z 295$ [M-H-298] $; 277$ $\left[\mathrm{M}-\mathrm{H}-298-\mathrm{H}_{2} \mathrm{O}\right]^{-}, 251\left[\mathrm{M}-\mathrm{H}-298-\mathrm{CO}_{2}\right]^{-}, 195$, and 171 were detected suggesting the presence of hydroxy-octadecadienoic acids derivative [38].

3.1.8. Miscellaneous Compounds. For L-ascorbic acid (1) and its isomers $(9,12,16,39,53,82,95$, and 101), a protonated pseudomolecular ion was observed at $m / z 177$ and $\mathrm{MS}^{2}$ ion at $m / z 133(\mathrm{M}+\mathrm{H}-44)^{+}$[13]. Moreover, compound 5 was suggested to be sucrose $\left(\mathrm{MS}^{1}\right.$ at $m / z 341[\mathrm{M}-\mathrm{H}]^{-}, \mathrm{MS}^{2}$ at $m$ / $z 179$ [M-H-glc. $\left.]^{-}, 161,131\right)$ [16]. It was previously reported that L-ascorbic acid and sucrose were identified in Hibiscus species as $H$. sabdariffa contains higher amount of ascorbic acid compared to orange and mango [44-46].

Oleuropein (2) showed a deprotonated pseudomolecular ion at $\mathrm{m} / z 539\left(\mathrm{MS}^{2} \text { at } \mathrm{m} / z 377 \text { [M-H-glc. }\right]^{-}$and 307 $\left[\mathrm{M}-\mathrm{H} \text {-glc. }-\mathrm{C}_{4} \mathrm{H}_{6} \mathrm{O}\right]^{-}$) [14]. It was previously reported that oleuropein was identified in Hibiscus [47]. Succinic acid (14) and its isomers $(17,52,66,70,73,83,87,90$, and 103) were recognized by comparing their $\mathrm{MS}^{2}$ fragmentation pattern with the reported data [22]. They showed a deprotonated molecular ion at $m / z 117$ and an intense fragment at $m / z 99$, attributed to the loss of water molecule [22], while compound 3 showed a protonated molecular ion at $m / z 119$ with
$\mathrm{MS}^{2}$ intense fragment ion at $m / z 101[\mathrm{M}+\mathrm{H}-18]^{+}$, attributed to the loss of water molecule $(-18 \mathrm{Da})$; thus, it was tentatively identified as succinic acid.

Hydroxycitric acid derivative (6) and butein chalcone (7) were identified by comparison with published data $[13,17]$, respectively. Hydroxycitric acid is the principal organic acid found in the calyces of Hibiscus according to [48]. Moreover, compound 38 was identified as a sesquiterpenoid derivative, gamma-eudesmol rhamnoside derivative $\left(\mathrm{MS}^{1}\right.$ at $\mathrm{m} / z 577$ $[\mathrm{M}-\mathrm{H}]^{-}, \mathrm{MS}^{2}$ at $m / z 439[\mathrm{M}-\mathrm{H}-138]^{-}$, corresponding to gamma-eudesmol, $293[\mathrm{M}-\mathrm{H}-138-146]^{-}$, corresponding to loss of rhamnose moiety $(-146 \mathrm{Da})$ ) [13]. N-feruloyltyramine (10), its isomer (60), and 22-dehydrocholesterol (77) were recognized by comparing their MS/MS fragmentation pattern with the reported data [13].

3.1.9. Unidentified Compounds. Finally, seven compounds $(44,61,80,81,88,91$, and 100) with the pseudomolecular ions $[\mathrm{M}-\mathrm{H}]^{-}$at $m / z 319$ and 877 and $[\mathrm{M}+\mathrm{H}]^{+}$at $m / z 274$, $274,399,515,805$, and 871 , respectively, could not be identified. Furthermore, of the 103 compounds identified, 7 compounds are unidentified; 46 compounds in $\mathrm{HdA}, 42$ compounds in HcA, and 25 compounds in HmA have been reported in the present study (Table 1). In conclusion, combination of accurate mass measurement and LC ability to separate isomeric compounds can be considered a powerful tool in the identification of polyphenol diversity in three species of the Hibiscus genus even in the absence of standards, but the stereochemical differentiation between the large number of isomers that were found in our species, for example, isomers of luteolin $C$-hexoside- $C$-pentoside, apigenin $C$-hexoside- $C$-pentoside, and cyanidin rutinoside, was not possible with our methodology.

3.2. Antioxidant Activity. It is well known that plant phenols and flavonoids in general are highly effective free radical scavengers and antioxidants. Thus, they are used for the prevention and cure of various disorders which are mainly associated with free radicals. Series of concentrations ranged from 5 to $320 \mu \mathrm{g} / \mathrm{mL}$ in methanol were used. The DPPH scavenging percentage of different extracts as well as ascorbic acid and $\mathrm{SC}_{50}$ values (the concentration required to scavenge DPPH by 50\%) are shown in Figures 2 and 3, respectively. HcA exhibited the highest antioxidant activity as indicated by its high DPPH scavenging percentage (65\%) at $320 \mu \mathrm{g} / \mathrm{mL}$ and low $\mathrm{SC}_{50}$ values $(111 \pm 1.5 \mu \mathrm{g} / \mathrm{mL})$. Its activity can be attributed to its contents of polyphenolic compounds such as phenolic acids, flavonoids, and anthocyanins (i.e., apigenin $C$-hexoside- $C$-pentoside, luteolin $C$ hexoside- $C$-pentoside, luteolin derivative, 4-hydroxybenzoic acid, tyrosol and peonidin derivative, and succinic acid). Unfortunately, both $\mathrm{HdA}$ and $\mathrm{HmA}$ displayed moderate antioxidant activities with $\mathrm{SC}_{50}=137.6 \pm 0.3$ and $135 \pm 0.5 \mu \mathrm{g} / \mathrm{mL}$, respectively, with ascorbic acid $\mathrm{SC}_{50}=14.2 \pm 0.5 \mu \mathrm{g} / \mathrm{mL}$ as standard.

During this work, many major anthocyanins were detected in LC-MS analysis of HcA (such as peonidin dirhamnoside, peonidin derivative, and peonidin-3- $(p-$ 


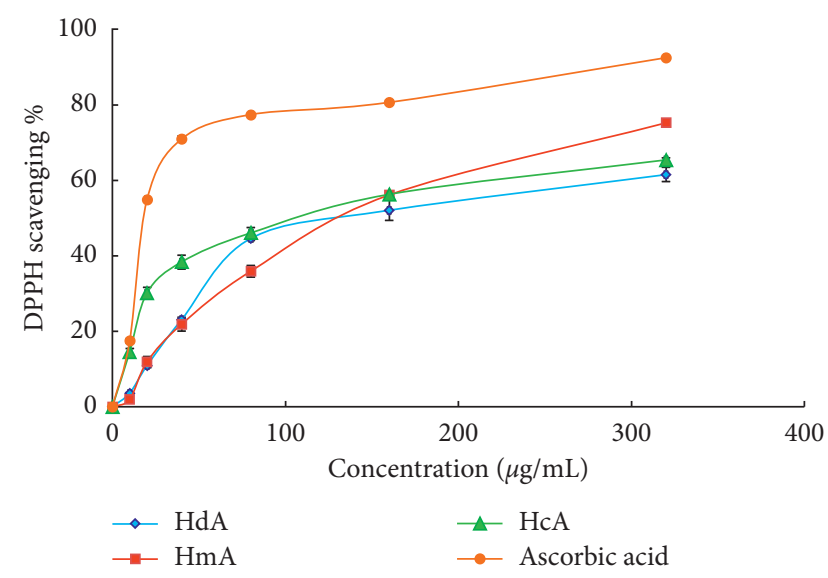

Figure 2: DPPH scavenging capacity of aqueous extracts of $\mathrm{Hi}$ biscus deflersii (HdA), H. micranthus (HmA), and H. calyphyllus (HcA).

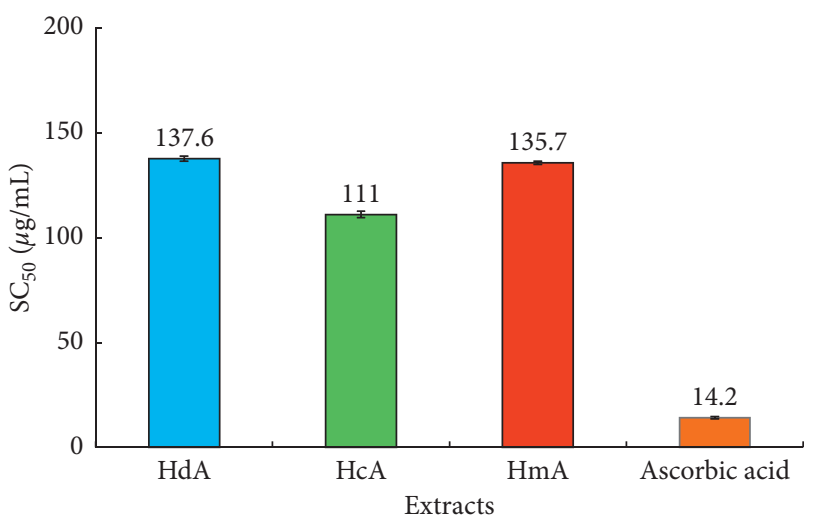

Figure 3: $\mathrm{SC}_{50}$ of antioxidant activity of Hibiscus deflersii (HdA), H. micranthus (HmA), and H. calyphyllus ( $\mathrm{HcA})$.

coumaroyl-glucoside)). According to [49], anthocyanin's high antioxidant activity was evidenced and is related to its structure, including the type, number, and position of the substituents in the flavylium cation. $3^{\prime}$-Hydroxyl group in cyanidin forms a catechol structure in the B ring, stabilizes the semiquinone radical, and forms a stable quinone that inhibits free radicals, such as DPPH, while $3^{\prime}, 5^{\prime}$-dihydroxyl group of delphinidin forms a pyrogallol structure in the B ring that has more delocalized electrons to stabilize the free radical generated in the medium. Ethyl acetate fraction of HcA, in a previous study, showed more significant antioxidant activity $\left(\mathrm{SC}_{50}=17.6 \pm 1.8 \mu \mathrm{g} / \mathrm{mL}\right)$ than the other extracts of HdA and HmA [5]. Many potential pathways for phenolic compounds to act as antioxidants were listed such as inhibiting free radical formation, peroxide decomposition, oxygen radical absorbance, free radical scavenging, suppression of singlet oxygen, increasing the levels of endogenous defenses, chelating of metal ions, and enzymatic inhibition [50,51]. There is a direct relation between the total polyphenolic content and the antioxidant activity [52] as polyphenolic compounds such as phenolic acids, flavonoids, and anthocyanins may be responsible for the antioxidant activity on a large proportion [51].
Correlation between radical scavenging ability, $\mathrm{SC}_{50}$ values, and the identified phenolic acids in LC-MS analysis is considerable as extracts with higher flavonoids and/or phenolics contents showed higher antioxidant activity and lower $\mathrm{SC}_{50}$ value. Salem et al. [53] stated in a previous study that flowers and leaves of $H$. rosa-sinensis, $H$. sabdariffa calyx extract, and $H$. platanifolius leaves extract possessed antioxidant activity that may be attributed to anthocyanins, flavonoids, and ascorbic acid content.

3.3. Cytotoxicity Assay. About $70 \%$ of death, in low- and middle-income countries, is caused by cancer [54]. For new effective anticancer drug discovery, screening of the cytotoxic activity of the plant extracts and natural products is necessary [55]. Edible plants are excellent resources of anticancer agents [56].

In our study, in vitro cytotoxic activity of the applied samples against tested cell lines using MTT assay and cisplatin as a positive standard showed a decrease in cell viability in dose-dependent manner as illustrated in Figure 4 and Table 2. Evaluation was based on $\mathrm{IC}_{50}$ values as follows: $\mathrm{IC}_{50} \leq 20 \mu \mathrm{g} / \mathrm{mL}$ highly active, $\mathrm{IC}_{50} 21-200 \mu \mathrm{g} / \mathrm{mL}$ moderately active, IC IC $_{50} 201-500 \mu \mathrm{g} / \mathrm{mL}$ weakly active, and $\mathrm{IC}_{50}>501 \mu \mathrm{g} / \mathrm{mL}$ inactive, which is in a good accordance with the American National Cancer Institute protocol [57].

In case of A-549 cell line, the cytotoxicity of the applied extracts was arranged as follows: $\mathrm{HdA}>\mathrm{HmA}>\mathrm{HcA}$. Unfortunately, HcA exhibited the weakest cytotoxic activity against A-549 cell line with $\mathrm{IC}_{50}$ of $113 \pm 3.4 \mu \mathrm{g} / \mathrm{mL}$ when compared to cisplatin, $7.53 \pm 3.8 \mu \mathrm{g} / \mathrm{mL}$. The higher activity of $\mathrm{HdA}\left(\mathrm{IC}_{50}=50 \pm 5.1 \mu \mathrm{g} / \mathrm{mL}\right)$ as a strong antioxidant may be attributed to the presence of major compounds such as butein flavonoid [58] and peonidin dipentoside anthocyanin according to Mahadevan et al. [44, 49, 59]. HdA is more cytotoxic to A-549 cells $\left(\mathrm{IC}_{50}=50 \pm 5.1 \mu \mathrm{g} / \mathrm{mL}\right.$ ) than $\mathrm{HmA}$ $\left(\mathrm{IC}_{50}=60.4 \pm 1.7 \mu \mathrm{g} / \mathrm{mL}\right)$ although both of them have nearly similar common major compounds, oleuropein and peonidin dipentoside.

As indicated by $\mathrm{IC}_{50}$ values, the cytotoxicity of tested samples against HCT-116 cell is arranged as follow: $\mathrm{HmA}>\mathrm{HcA}>\mathrm{HdA}$. A close cytotoxic effect on HCT-116 cell line was shown by HdA and HcA $\left(\mathrm{IC}_{50}=96 \pm 3.2\right.$ and $92.9 \pm 4.1 \mu \mathrm{g} / \mathrm{mL}$, respectively). The cytotoxic activity of $\mathrm{HmA}$ may be attributed to $\mathrm{N}$-feruloyltyramine as it was reported as a cytotoxic agent in a previous study [60], and this cytotoxic activity may be enhanced by the presence of ascorbic acid according to [61, 62].

Our results are in agreement with those reported for the cytotoxicity of flavonoids, phenolic acids, and terpenes content which are major constituents identified in HmA in that study [63-66]. In a previous study, different extracts (ethyl acetate, chloroform, petroleum ether) of HcA, HdA, and $\mathrm{HmA}$ showed strong anticancer property against human hepatocellular carcinoma (HepG2) and human breast carcinoma (MCF-7) cell lines [63]. Leaves, calyx, and stem extracts of other species of Hibiscus (such as $H$. sabdariffa, $H$. rosa-sinensis, $H$. micranthus, $H$. vitifolius, and $H$. syriacus) have shown promising cytotoxic activity against many 


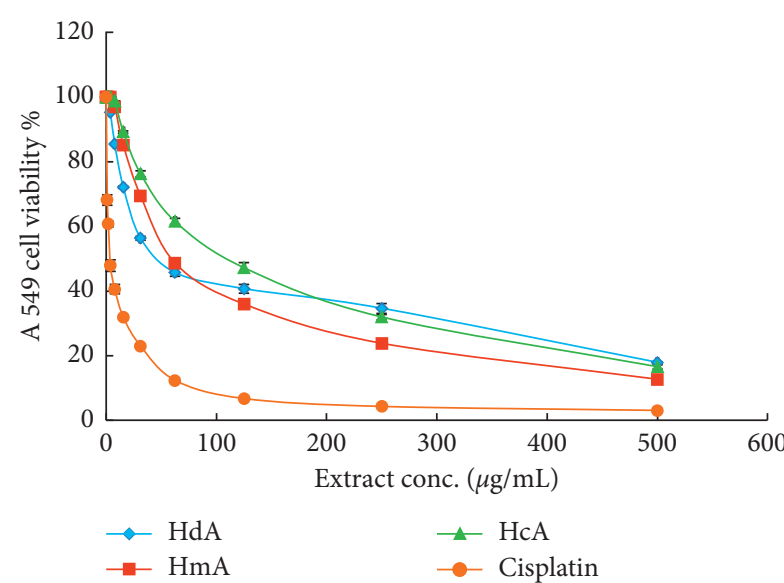

(a)

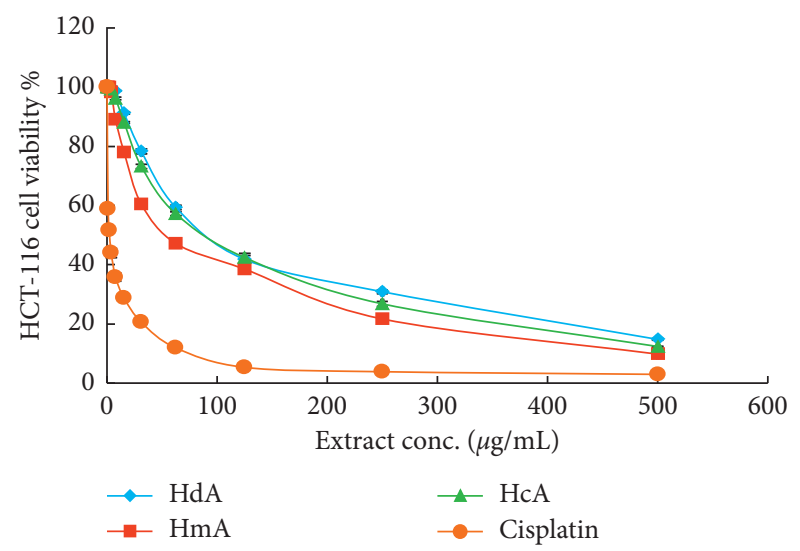

(b)

Figure 4: In vitro cytotoxic activity of aqueous extracts of tested Hibiscus deflersii (HdA), H. micranthus (HmA), and H. calyphyllus (HcA) against A-549 (a) and HCT-116 (b) cell lines.

TABLE 2: $\mathrm{IC}_{50}$ of tested aqueous extracts of Hibiscus deflersii (HdA), H. micranthus (HmA), and H. calyphyllus (HcA) against A-549 and HTC-116 cell lines.

\begin{tabular}{lcc}
\hline & $\mathrm{IC}_{50}(\mu \mathrm{g} / \mathrm{mL})$ & \\
Tested extracts & A-549 cell line & HCT-116 cell line \\
\hline HdA & $50 \pm 5.1$ & $96 \pm 3.2$ \\
HcA & $113 \pm 3.4$ & $92.9 \pm 2.8$ \\
HmA & $60.4 \pm 1.7$ & $56 \pm 1.9$ \\
Cisplatin & $7.53 \pm 3.8$ & $2.43 \pm 4.1$ \\
\hline
\end{tabular}

These are the means of three determinations. The data are presented as $\mu \mathrm{g} / \mathrm{mL}$.

cancer cells such as breast, lung, and human leukemia cells (HL-60) and liver cancer cell lines, and they showed potent cytotoxic activity against human lung cancer cell line (A549) that may be attributed to the presence of flavonoids, tannins, triterpenes, phenols, steroids $[67,68]$, polyphenolic compounds, such as protocatechuic acid, anthocyanins such as delphinidin-3-sambubioside, and myristic acid and uncarinic acid A $[45,48,52]$.

3.4. Antidiabetic Assay. Diabetes mellitus (DM) is a persistent disorder that is incurable due to the deficiency of insulin that affects $10 \%$ of the population. It is expected to extend the number of diabetic individuals to 230 million in 2025. There are many side effects for drugs currently used in DM treatment, so herbal medicines are highly recommended for the treatment of diabetes instead of other synthetic drugs [3]. Since ancient times, DM has been treated orally using folklore medicine with several medicinal plants or their extracts [65].

In the present study, in vitro $\alpha$-amylase inhibitory activity of the applied samples, evaluated using different doses $(7.81-1000 \mu \mathrm{g} / \mathrm{mL})$, showed significant inhibition of carbohydrate hydrolyzing enzymes ( $\alpha$-amylase) in dose-dependent manner as illustrated in Figures 5 and 6 . HdA exhibited higher $\alpha$-amylase inhibitory activity $(78.85 \pm 1.8 \%$,
$\left.\mathrm{IC}_{50}=56.22 \pm 1.9 \mu \mathrm{g} / \mathrm{mL}\right)$ than that of $\mathrm{HcA}(68.0 \pm 0.4 \%$, $\left.\mathrm{IC}_{50}=103.9 \pm 1.5 \mu \mathrm{g} / \mathrm{mL}\right)$ and $\mathrm{HmA}\left(63.58 \pm 1.9 \%, \mathrm{IC}_{50}=\right.$ $149.07 \pm 2.1 \mu \mathrm{g} / \mathrm{mL}$ ) against acarbose standard with $\mathrm{IC}_{50}=$ $34.71 \pm 0.7 \mu \mathrm{g} / \mathrm{mL}$. The higher activity of $\mathrm{HdA}\left(\mathrm{IC}_{50}=56.22 \pm\right.$ $1.9 \mu \mathrm{g} / \mathrm{mL}$ ) may be attributed to the presence of oleuropein. Jemai et al. [69] previously reported that oleuropein prevents some metabolic diseases related to oxidative stress such as diabetes, hypercholesterolemia associated with diabetes, and cardiovascular complications which are very predominant in diabetics, due to its hypoglycemic activity as it enhances peripheral glucose uptake or insulin release and stimulates the synthesis of liver glycogen through its antioxidant power.

In agreement with our results, other studies have reported that aerial parts of HdA were used as potential antidiabetics due to the presence of flavonoids [70]. The flowers and fruits of HmA were found effective in diabetes [71]. The reported hypoglycemic activity of methanol leaf extract of $H$. sabdariffa and $H$. rosa-sinensis and flowers extract of $H$. vitifolius and $H$. tiliaceus may refer to the presence of flavonoids, phenols, tannins, alkaloids, and saponins $[48,65,72]$.

3.5. Antiobesity Activity. Overweight and obesity are chronic disorders that are considered as a growing issue influencing both adults and children. Obesity is defined as irregular or excessive fat accumulation caused by the imbalance between energy intake and expenditure. The vast majority of metabolic disorders such as cardiovascular disease, dyslipidemia, hypertension, and diabetes may be due to obesity or overweight $[45,73]$. The inhibition of the digestion and absorption of dietary fats is a promising remedy for obesity. Natural products are preferable to obesity drugs such as orlistat which have many side effects (i.e., development of cardiovascular problems, restlessness, sleeping disorder, and stomach pain) [73].

Results of the antiobesity activity of the three Hibiscus species aqueous extracts grown in Saudi Arabia using in vitro 


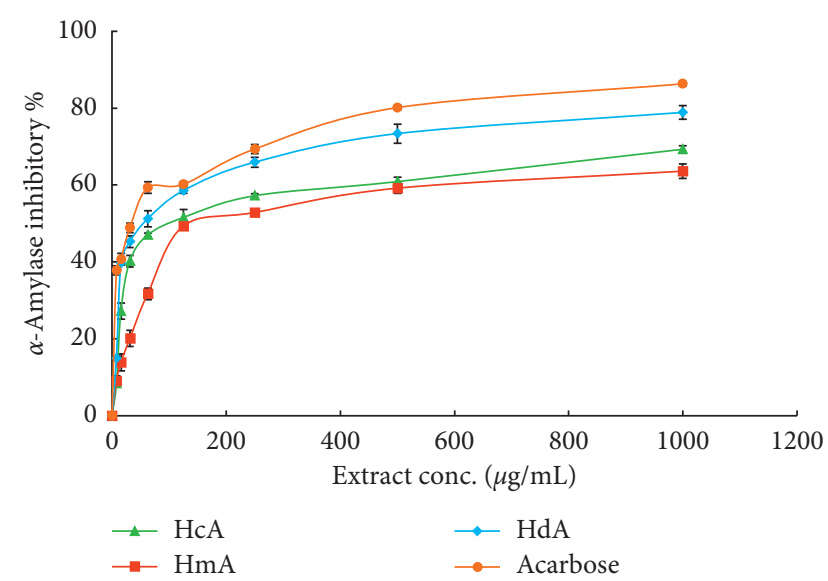

FIGURE 5: In vitro $\alpha$-amylase inhibitory activity of aqueous extracts of tested Hibiscus deflersii (HdA), $H$. micranthus (HmA), and $H$. calyphyllus (HcA).

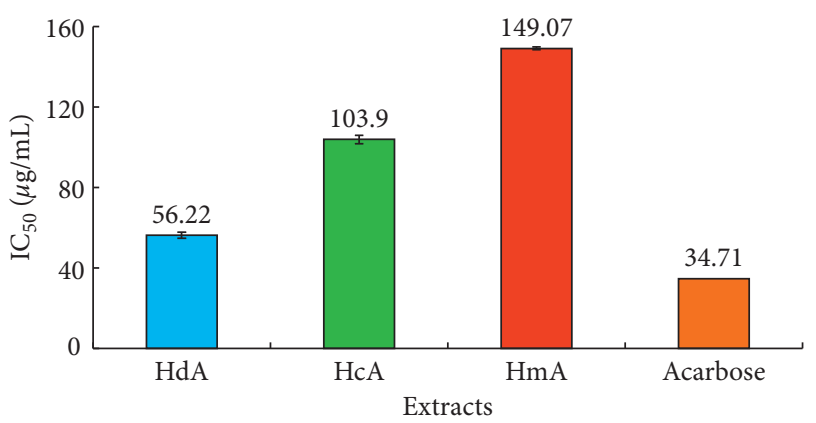

FIGURE 6: IC $_{50}$ of $\alpha$-amylase inhibitory activity of Hibiscus deflersii (HdA), H. micranthus (HmA), and H. calyphyllus (HcA) aqueous extracts and standard acarbose.

pancreatic lipase inhibitory assay are shown in Figure 7 and Table 3. HdA exhibited higher inhibitory activity than the $\mathrm{HmA}$ and $\mathrm{HcA}$ with $\mathrm{IC}_{50}$ of $95.45 \pm 1.9,107.7 \pm 1.5$, and $>1000 \mu \mathrm{g} / \mathrm{mL}$, respectively, comparable with orlistat $\left(\mathrm{IC}_{50}=23.8 \pm 0.7 \mu \mathrm{g} / \mathrm{mL}\right)$ as standard. Lipase inhibitory activity of HdA may be attributed to the presence of anthocyanins (peonidin-3-( $p$-coumaroyl-glucoside), peonidin dirhamnoside, peonidin glucoside feruloyl glucuronide, peonidin dipentoside, malvidin derivative, and malvidin-3$O$-glucoside derivative) and organic acids (such as succinic, ascorbic, and 4-hydroxybenzoic acid). It was reported that polyphenol compounds such as anthocyanins [74] and organic acids [75] are responsible for the antiobesity activity. Da-Costa-Rocha et al. [48] stated that Hibiscus extract (or tea) may help in weight loss as antiobesity agent due to its effects on fat absorption-excretion, inhibition of the activity of $\alpha$-amylase, starch absorption, and blocking sugars. Moreover, aqueous extract of Hibiscus species showed a powerful inhibition of triglyceride accumulation as whole extract was more active than isolated polyphenols.

In a previous study, aqueous extract of $H$. sabdariffa (with anthocyanins being major compounds) exhibited many potential antiobesity mechanisms, including

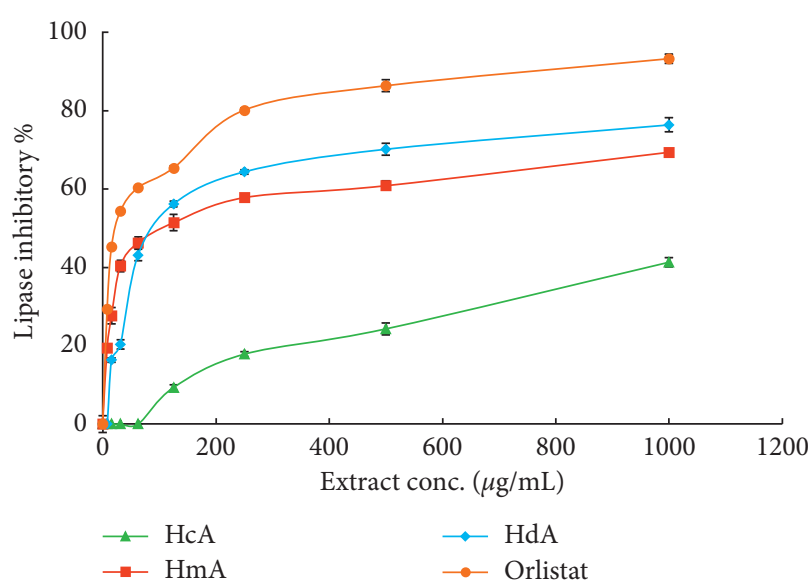

FIGURE 7: In vitro lipase inhibitory activity of aqueous extracts of tested Hibiscus deflersii (HdA), $H$. micranthus (HmA), and $H$. calyphyllus (HcA) in comparison with orlistat standard.

TABLE 3: $\mathrm{IC}_{50}$ of tested aqueous extracts of Hibiscus deflersii (HdA), H. micranthus (HmA), and H. calyphyllus (HcA) against pancreatic lipase enzyme.

\begin{tabular}{lc}
\hline & $\mathrm{IC}_{50}(\mu \mathrm{g} / \mathrm{mL})$ \\
\hline Tested extracts & \\
HdA & $95.45 \pm 1.9$ \\
$\mathrm{HcA}$ & $>1000$ \\
HmA & $107.7 \pm 1.5$ \\
Orlistat standard & $23.8 \pm 0.7$ \\
\hline
\end{tabular}

These are the means of three determinations. The data are presented as $\mu \mathrm{g} / \mathrm{mL}$.

antihyperglycemic activity, reduction in plasma cholesterol level, inhibition of gastric and pancreatic lipase enzymes, thermogenesis stimulation, inhibition of lipid droplet accumulation in fat cells, and fatty acid synthase inhibition [76]. Drinking a cup of Hibiscus tea after meals can reduce the absorption of dietary carbohydrates and assist in weight loss [45].

\section{Conclusion}

Phenolic compounds, flavonoids, and anthocyanins were identified in three different Hibiscus species using UPLCESI-MS/MS analysis. HcA showed the highest in vitro antioxidant activity compared with other tested extracts, and this activity can be attributed to its contents of polyphenolic compounds such as apigenin $C$-hexoside- $C$-pentoside, luteolin $C$-hexoside- $C$-pentoside, luteolin derivative, 4hydroxybenzoic acid, and tyrosol and peonidin derivative in addition to presence of anthocyanin contents such as peonidin dirhamnoside, peonidin derivative, and peonidin3-( $p$-coumaroyl-glucoside). HdA showed the most potent effect on human lung carcinoma (A-549) cell line, which may be attributed to the presence of major compounds such as butein flavonoid and peonidin dirhamnoside and peonidin dipentoside anthocyanins, with the highest activities as antidiabetic (due to oleuropein presence) and as antiobesity (may be attributed to the presence of major anthocyanins 
such as peonidin-3-( $p$-coumaroyl-glucoside), peonidin dirhamnoside, and peonidin glucoside feruloyl glucuronide in addition to organic acids (such as succinic, ascorbic, and 4-hydroxybenzoic acid)). The results recommend that HdA need further studies for the possible use as anticancer, antidiabetic, and antiobesity agent as it might be a natural alternative remedy and nutritional policy for diabetes and obesity treatment without negative side effects. Isolation of the bioactive phytochemical from the $\mathrm{HcA}, \mathrm{HmA}$, and $\mathrm{HdA}$ and estimation of their biological effects are recommended in further studies.

\section{Data Availability}

The data used to support the findings of this study are included within the article.

\section{Conflicts of Interest}

The authors declare that they have no conflicts of interest.

\section{Authors' Contributions}

All authors made considerable contributions to the manuscript. HA, ME, MA, RA, and SA designed the study. ME, $\mathrm{WH}, \mathrm{HA}, \mathrm{SA}, \mathrm{MA}$, and RA performed the experiments. ME, SA, HA, and WH interpreted the results. ME, HA, and SA wrote the manuscript. All authors revised the manuscript and approved it for publication.

\section{Acknowledgments}

The authors thank Prof. Dr. Mohamed Yousef, Pharmacognosy Department, College of Pharmacy, King Saud University (KSU), for the plant identification. This research was carried out with personal funds from the authors.

\section{Supplementary Materials}

Table S1: metabolites identified in the aqueous extract of three different Hibiscus species: Hibiscus deflersii (HdA), $H$. micranthus (HmA), and H. calyphyllus (HcA), using UPLCESI-MS in negative and positive ionization modes. Table S2: $\mathrm{IC}_{50}$ of tested aqueous Hibiscus extracts of Hibiscus deflersii (HdA), H. micranthus (HmA), and H. calyphyllus (HcA) against A-549 and HTC-116 cell lines. The data are presented as $\mu \mathrm{g} / \mathrm{mL}$. Table S3: $\mathrm{IC}_{50}$ of tested aqueous Hibiscus extracts of Hibiscus deflersii (HdA), H. micranthus (HmA), and $H$. calyphyllus (HcA) against pancreatic lipase enzyme. The data are presented as $\mu \mathrm{g} / \mathrm{mL}$. (Supplementary Materials)

\section{References}

[1] C. S. Kılıç, S. Aslan, and M. Kartal MandCoşkun, "Fatty acid composition of Hibiscus trionum L. (Malvaceae)," Journal of Natural Products, vol. 5, pp. 65-69, 2011.

[2] N. Vasudeva and S. K. Sharma, "Biologically active compounds from the Genus Hibiscus," Pharmaceutical Biology, vol. 46, no. 3, pp. 145-153, 2008.
[3] S. Venkatesh, J. Thilagavathi, and D. Shyam sundar, "Antidiabetic activity of flowers of Hibiscus rosasinensis," Fitoterapia, vol. 79, no. 2, pp. 79-81, 2008.

[4] L. B. Vinh, N. T. M. Nguyet, C. D. Thanh et al., "Chemical constituents of Vietnamese mangrove Hibiscus tiliaceus with antioxidant and alpha-glucosidase inhibitory activity," Natural Product Research, pp. 1-6, 2019.

[5] N. A. Siddiqui, H. M. Al-Yousef, T. A. Alhowiriny et al., "Concurrent analysis of bioactive triterpenes oleanolic acid and $\beta$-amyrin in antioxidant active fractions of Hibiscus calyphyllus, Hibiscus deflersii and Hibiscus micranthus grown in Saudi Arabia by applying validated HPTLC method," Saudi Pharmaceutical Journal, vol. 26, no. 2, pp. 266-273, 2018.

[6] W. H. B. Hassan, S. Abdelaziz, and H. M. Yousef, "Chemical composition and biological activities of the aqueous fraction of Parkinsonea aculeata L. growing in Saudi Arabia," Arabian Journal of Chemistry, vol. 12, no. 3, pp. 377-387, 2019.

[7] G. C. Yen and P. D. Duh, "Scavenging effect of methanolic extracts of peanut hulls on free-radical and active-oxygen species," Journal of Agricultural and Food Chemistry, vol. 42, no. 3, pp. 629-632, 1994.

[8] I. Gülçin, Öüfre Küfrevioğlu, M. Oktay, and M. E. Büyükokuroğlu, "Antioxidant, antimicrobial, antiulcer and analgesic activities of nettle (Urtica dioica L.)," Journal of Ethnopharmacology, vol. 90, no. 2-3, pp. 205-215, 2004.

[9] S. M. Gomha, T. A Salah, and A. O. Abdelhamid, "Synthesis, characterization, and pharmacological evaluation of some novel thiadiazoles and thiazoles incorporating pyrazole moiety as anticancer agents," Monatshefte für Chemie Chemical Monthly, vol. 146, no. 1, pp. 149-158, 2015.

[10] T. Mosmann, "Rapid colorimetric assay for cellular growth and survival: application to proliferation and cytotoxicity assays," Journal of Immunological Methods, vol. 65, no. 1-2, pp. 55-63, 1983.

[11] M. B. Narkhede, P. V. Ajimire, A. E. Wagh, M. Mohan, and A. T. Shivashanmugam, "In vitro antidiabetic activity of Caesalpina digyna (R.) methanol root extract," Asian Journal of Plant Science and Research, vol. 1, no. 2, pp. 101-106, 2011.

[12] Y. S. Kim, Y. M. Lee, H. Kim, J. Kim, D. S. Jang, and J. H. Kim, "Anti-obesity effect of Morus bombycis root extract: anti-lipase activity and lipolytic effect," Journal of Ethnopharmacology, vol. 130, no. 3, pp. 621-624, 2010.

[13] FooDB, “The Metabolomics Innovation Centre," 2019, https://foodb.ca/.

[14] H. Zemmouri, S. Ammar, A. Boumendjel, M. Messarah, and A. El Feki, "Chemical composition and antioxidant activity of Borago officinalis L. leaf extract growing in Algeria," Arabian Journal of Chemistry, vol. 12, no. 8, pp. 1954-1963, 2019.

[15] M. Lambert, E. Meudec, A. Verbaere et al., "A highthroughput UHPLC-QqQ-MS method for polyphenol profiling in rosé wines," Molecules, vol. 20, no. 5, pp. 7890-7914, 2015.

[16] S. Moco, Metabolomics Technologies Applied to the Identification of Compounds in Plants: A Liquid ChromatographyMass Spectrometry-Nuclear Magnetic Resonance Perspective over the Tomato Fruit, Ph.D. thesis, Wageningen University, Wageningen, The Netherlands, 2007.

[17] I. M. Shi, M. S. Ali-Shtayeh, R. M. Jamous, and D. ArráezRomán, "HPLC-DAD-ESI-MS/MS screening of bioactive components from Rhus coriaria L. (Sumac) fruits," Food Chemistry, vol. 166, pp. 179-191, 2015.

[18] P. Efferth, A. Vallverdú-Queralt, M. Martínez-Huélamo et al., "A comprehensive characterisation of beer polyphenols by 
high resolution mass spectrometry (LC-ESI-LTQ-OrbitrapMS)," Food Chemistry, vol. 169, pp. 336-343, 2015.

[19] E. Émile Nicoué, "Identification des anthocyanes de deux espèces de bleuets sauvages du Québec "Vaccinium angustifolium" et "Vaccinium myrtilloïdes" et évaluation de leur potentiel antioxydant," Université Laval Quebec, Canada, Doctoral dissertation, 2010.

[20] Q. Chang and Y. S. Wong, "Identification of flavonoids in hakmeitau beans (Vigna sinensis) by high-performance liquid chromatography-electrospray mass spectrometry (LC-ESI/ MS)," Journal of Agricultural and Food Chemistry, vol. 52, no. 22, pp. 6694-6699, 2004.

[21] R. O. Bakr, S. A. E. H. Mohamed, and N. Ayoub, "Phenolic profile of Centaurea aegyptiaca L. growing in Egypt and its cytotoxic and antiviral activities," African Journal of Traditional, Complementary and Alternative Medicines, vol. 13, no. 6, pp. 135-143, 2016.

[22] O. Al Kadhi, A. Melchini, R. Mithen, and S. Saha, "Development of a LC-MS/MS method for the simultaneous detection of tricarboxylic acid cycle intermediates in a range of biological matrices," Journal of Analytical Methods in Chemistry, vol. 2017, Article ID 5391832, 12 pages, 2017.

[23] F. Ferreres, C. Grosso, A. Gil-Izquierdo, P. Valentão, and C. Azevedo, "HPLC-DAD-ESI/MSn analysis of phenolic compounds for quality control of Grindelia robusta Nutt. and bioactivities," Journal of Pharmaceutical and Biomedical Analysis, vol. 94, pp. 163-172, 2014.

[24] A. Sinela, N. Rawat, C. Mertz, N. Achir, and M. Fulcrand HandDornier, "Anthocyanins degradation during storage of Hibiscus sabdariffa extract and evolution of its degradation products," Food Chemistry, vol. 214, pp. 234-241, 2017.

[25] S. Ammar, M. Del Mar Contreras, O. Belguith-Hadrich, A. Segura-Carretero, and M. Bouaziz, "Assessment of the distribution of phenolic compounds and contribution to the antioxidant activity in Tunisian fig leaves, fruits, skins and pulps using mass spectrometry-based analysis," Food and Function, vol. 6, no. 12, pp. 3663-3677, 2015.

[26] D. Barreca, E. Bellocco, C. Caristi, and U. Leuzzi, "Flavonoid profile and radical-scavenging activity of Mediterranean sweet lemon (Citrus limetta risso) juice," Food Chemistry, vol. 129, no. 2, pp. 417-422, 2011.

[27] R. M. IbrahimLin, A. M. El-Halawany, D. O. Saleh et al., "HPLC-DAD-MS/MS profiling of phenolics from Securigera securidaca flowers and its anti-hyperglycemic and antihyperlipidemic activities," Revista Brasileira de Farmacognosia, vol. 25, no. 2, pp. 134-141, 2015.

[28] I. C. Rodríguez-Medina, R. Beltrán-Debón, V. M. Molina et al., "Direct characterization of aqueous extract of Hibiscus sabdariffa using HPLC with diode array detection coupled to ESI and ion trap MS," Journal of Separation Science, vol. 32, no. 20, pp. 3441-3448, 2009.

[29] M. Simirgiotis, J. Benites, and C. Areche, "Antioxidant capacities and analysis of phenolic compounds in three endemic nolana species by HPLC-PDA-ESI-MS," Molecules, vol. 20, no. 6, pp. 11490-11507, 2015.

[30] U. Shin, "Negative atmospheric pressure chemical ionisation low-energy collision activation mass spectrometry for the characterisation of flavonoids in extracts of fresh herbs," Journal of Chromatography A, vol. 902, no. 2, pp. 369-379, 2000.

[31] V. Spínola, J Pinto, and P. C. Castilho, "Identification and quantification of phenolic compounds of selected fruits from Madeira Island by HPLC-DAD-ESI-MSn and screening for their antioxidant activity," Food Chemistry, vol. 173, pp. 1430, 2015.

[32] C. A. Ledesma-Escobar, F. Priego-Capote, and M. D. Luque De Castro, "Characterization of lemon (Citrus limon) polar extract by liquid chromatography-tandem mass spectrometry in high resolution mode," Journal of Mass Spectrometry, vol. 50, no. 11, pp. 1196-1205, 2015.

[33] M. B. Hossain, D. K. Rai, N. P. Brunton, A. B. Martin-Diana, and B. R. Catherine, "Characterization of phenolic composition in lamiaceae spices by LC-ESI-MS/MS," Journal of Agricultural and Food Chemistry, vol. 58, no. 19, pp. 1057610581, 2010.

[34] R. Colombo, J. H. Yariwake, and M. McCullagh, "Study of $\mathrm{C}$-and O-glycosylflavones in sugar cane extracts using liquid chromatography-exact mass measurement mass spectrometry," Journal of the Brazilian Chemical Society, vol. 19, no. 3, pp. 483-490, 2008.

[35] R. Stein-Chisholm, J. Beaulieu, and C. Grimm, "LC-MS/MS and UPLC-UV evaluation of anthocyanins and anthocyanidins during rabbiteye blueberry juice processing," Beverages, vol. 3, no. 4, p. 56, 2017.

[36] A. Brito, A. Gattuso, J. Ramirez, C. Areche, B. Sepúlveda, and M. Simirgiotis, "HPLC-UV-MS profiles of phenolic compounds and antioxidant activity of fruits from three citrus species consumed in northern Chile," Molecules, vol. 19, no. 11, pp. 17400-17421, 2014.

[37] M. P. Rodríguez-Rivera, E. Lugo-Cervantes, and P. Winterhalter, "Metabolite profiling of polyphenols in peels of Citrus limetta Risso by combination of preparative highspeed countercurrent chromatography and LC-ESI-MS/MS," Food Chemistry, vol. 158, pp. 139-152, 2014.

[38] M. A. Farag, S. T. Sakna, N. M. El-Fiky, M. M. Shabana, and L. A. Wessjohann, "Phytochemical, antioxidant and antidiabetic evaluation of eight Bauhinia L. species from Egypt using UHPLC-PDA-qTOF-MS and chemometrics," Phytochemistry, vol. 119, pp. 41-50, 2015.

[39] A. A. Chernonosov, E. A. Karpova, and E. M. Lyakh, "Identification of phenolic compounds in Myricaria bracteata leaves by high-performance liquid chromatography with a diode array detector and liquid chromatography with tandem mass spectrometry," Revista Brasileira de Farmacognosia, vol. 27, no. 5, pp. 576-579, 2017.

[40] W. Y. Sayadi, J. S. Jin, Y. A. Cho et al., "Determination of polyphenols in three Capsicum annuum L. (bell pepper) varieties using high-performance liquid chromatographytandem mass spectrometry: their contribution to overall antioxidant and anticancer activity," Journal of Separation Science, vol. 34, no. 21, pp. 2967-2974, 2011.

[41] K. Ablajan, Z. Abliz, X.-Y. Shang, J.-M. He, and R.-P. Zhang, "Structural characterization of flavonol 3,7-di-O-glycosides and determination of the glycosylation position by using negative ion electrospray ionization tandem mass spectrometry," Journal of Mass Spectrometry, vol. 41, no. 3, pp. 352-360, 2006.

[42] R. Wang, M. Ye, H. Guo, K. Bi, and D.-a. Guo, "Liquid chromatography/electrospray ionization mass spectrometry for the characterization of twenty-three flavonoids in the extract of Dalbergia odorifera," Rapid Communications in Mass Spectrometry, vol. 19, no. 11, pp. 1557-1565, 2005.

[43] A. Andrade, R. Heydari, Z. Talebpour et al., "Study of new extraction methods for separation of anthocyanins from red grape skins: analysis by HPLC and LC-MS/MS," Journal of Liquid Chromatography \& Related Technologies, vol. 31, no. 17, pp. 2686-2703, 2008. 
[44] N. Mahadevan and P. Kamboj, "Hibiscus sabdariffa Linn.-an overview," Natural Product Radiance, vol. 8, no. 1, pp. 77-83, 2009.

[45] P. Singh, M. Khan, and H. Hailemariam, "Nutritional and health importance of Hibiscus sabdariffa: a review and indication for research needs," Journal of Nutritional Health \& Food Engineering, vol. 6, no. 5, Article ID 00212, 2017.

[46] P. K. Wong, S. Yusof, H. M. Ghazali, and Y. B. Che Man, "Physico-chemical characteristics of roselle (Hibiscus sabdariffa L.)," Nutrition \& Food Science, vol. 32, no. 2, pp. 68-73, 2002.

[47] J. Bouaziz, X. Zhang, J. Zhang, M. Li, and T. Chen, "Minor compounds of the high purity salvianolic acid B freeze-dried powder from Salvia miltiorrhiza and antibacterial activity assessment," Natural Product Research, vol. 32, no. 10, pp. 1198-1202, 2018.

[48] I. Da-Costa-Rocha, B. Bonnlaender, H. Sievers, I. Pischel, and M. Heinrich, Hibiscus sabdariffa L. - A Phytochemical and Pharmacological Review, Vol. 165, Elsevier Ltd., Amsterdam, Netherlands, 2014.

[49] L. G. Maciel, M. A. V. do Carmo, L. Azevedo et al., "Hibiscus sabdariffa anthocyanins-rich extract: chemical stability, in vitro antioxidant and antiproliferative activities," Food and Chemical Toxicology, vol. 113, pp. 187-197, 2018.

[50] M. N. Moraes, G. L. Zabot, J. M. Prado, and M. A. A. Meireles, "Obtaining antioxidants from botanic matrices applying novel extraction techniques," Food and Public Health, vol. 3, no. 4, pp. 195-214, 2013.

[51] A. Obouayeba, N. Djyh, S. Diabate et al., "Phytochemical and antioxidant activity of roselle (Hibiscus sabdariffa L.) petal extracts," Research Journal of Pharmaceutical, Biological and Chemical Sciences, vol. 5, no. 2, pp. 1453-1465, 2014.

[52] S. Abdelhamid, O. Martí n-Belloso, Y.-S. Park et al., "Comparison of some biochemical characteristics of different citrus fruits," Food Chemistry, vol. 74, no. 3, pp. 309-315, 2001.

[53] M. Z. M. Salem, J Olivares-Pérez, and A. Z. M. Salem, "Studies on biological activities and phytochemicals composition of Hibiscus species-a review," Life Science Journal, vol. 11, no. 5, pp. 1-8, 2014.

[54] V. Kim, F. W. Fokou, O. Karaosmanoğlu, V. P. Beng, and H. Sivas, "Cytotoxicity of the methanol extracts of Elephantopus mollis, Kalanchoe crenata and 4 other Cameroonian medicinal plants towards human carcinoma cells," BMC Complementary and Alternative Medicine, vol. 17, no. 1, 2017.

[55] L. K. Wasiman, J. O. Midiwo, V. M. Masila et al., "Cytotoxicity of 91 Kenyan indigenous medicinal plants towards human CCRF-CEM leukemia cells," Journal of Ethnopharmacology, vol. 179, pp. 177-196, 2016.

[56] H.-H. Qian, H.-P. Huang, C.-C. Huang, and J.-H. Chen, "Hibiscus polyphenol-rich extract induces apoptosis in human gastric carcinoma cells via p53 phosphorylation and p38 MAPK/FasL cascade pathway," Molecular Carcinogenesis, vol. 43, no. 2, pp. 86-99, 2005.

[57] T. Srisawat, P. Chumkaew, W. Heed-Chim, Y Sukpondma, and K. Kanokwiroon, "Phytochemical screening and cytotoxicity of crude extracts of Vatica diospyroides symington type LS," Tropical Journal of Pharmaceutical Research, vol. 12, no. 1, pp. 71-76, 2013.

[58] Y. Luque de Castro, C. Ma, M. Qian, Z. Wen, and H. Jing, "Butein induces cell apoptosis and inhibition of cyclooxygenase-2 expression in A549 lung cancer cells," Molecular Medicine Reports, vol. 9, no. 2, pp. 763-767, 2014.
[59] C.-H. Che Man, C.-C. Huang, C.-H. Hung, F.-Y. Yao, C.-J. Wang, and Y.-C. Chang, "Delphinidin-rich extracts of Hibiscus sabdariffa L. trigger mitochondria-derived autophagy and necrosis through reactive oxygen species in human breast cancer cells," Journal of Functional Foods, vol. 25, pp. 279-290, 2016.

[60] J.-J. Wong, S.-Y. Huang, C.-Y. Duh, I.-S. Chen, T.-C. Wang, and H.-Y. Fang, "A new cytotoxic amide from the stem wood of Hibiscus tiliaceus," Planta Medica, vol. 72, no. 10, pp. 935-938, 2006.

[61] C. M. Narsu, U. Wagnera, B. Kolsterb, P. E. Andreottic, D Krebsa, and H. W. Brucknerd, "Ascorbic acid (vitamin C) improves the antineoplastic activity of doxorubicin, cispiatin, and paclitaxel in human breast carcinoma cells in vitro," Cancer Letters, vol. 103, no. 2, pp. 183-189, 1996.

[62] H. Sakagami, K. Satoh, H. Ohata et al., "Relationship between ascorbyl radical intensity and apoptosis-inducing activity," Anticancer Res, vol. 16, no. 5, pp. 2635-2644, 1996.

[63] P. Segura-Carretero, H. M. Al-Yousef, N. A. Siddiqui et al., "Anticancer activity and concurrent analysis of ursolic acid, $\beta$-sitosterol and lupeol in three different Hibiscus species (aerial parts) by validated HPTLC method," Saudi Pharmaceutical Journal, vol. 26, no. 7, pp. 1060-1067, 2018.

[64] R. Jain, R Arora, and S. Jain, "Chemical constituents and bioactivity studies of Hibiscus Mucranthus Linn," Indian Journal of Pharmaceutical Sciences, vol. 59, no. 2, p. 91, 1997.

[65] K. A. Kumar, S. R. Setty, and L. Narsu, "Pharmacognostic and phytochemical investigations of stems of Hibiscus micranthus Linn," Pharmacognosy Journal, vol. 2, no. 15, pp. 21-30, 2010.

[66] R. M. Rosa, M. I. S. Melecchi, and R. D. C. Halmenschlager, J. Saffi and A. L. L. D. Ramos, Antioxidant and antimutagenic properties of Hibiscus tiliaceus L. methanolic extract," Journal of Agricultural and Food Chemistry, vol. 54, no. 19, pp. 7324-7330, 2006.

[67] T. Lamuela-Raventós, "Comparative pharmacological study of aerial parts and roots of ethanolic extract of Hibiscus micranthus Linn," Journal of Medical Pharmaceutical and allied Sciences, vol. 1, pp. 581-587, 2017.

[68] L.-S. Shi, C.-H. Wu, T.-C. Yang, C.-W. Yao, H.-C. Lin, and W.-L. Chang, "Cytotoxic effect of triterpenoids from the root bark of Hibiscus syriacus," Fitoterapia, vol. 97, pp. 184-191, 2014.

[69] H. Jemai, A. E. L. Feki, and S. Sayadi, "Antidiabetic and antioxidant effects of hydroxytyrosol and oleuropein from olive leaves in alloxan-diabetic rats," Journal of Agricultural and Food Chemistry, vol. 57, no. 19, pp. 8798-8804, 2009.

[70] S. Lakshman, A. Jyothi, V. Mounica, A. R. Kumar, S. Rathinam, and K. Rajesh, "Antidiabetic activity of methonolic extract of in streptozotocin induced diabetic rats Hibiscus deflersii," International Research Journal of Pharmaceutical and Applied Sciences, vol. 4, no. 1, pp. 1-3, 2014.

[71] H. Kakrani, B. H. Kakrani, and A. K. Saluja, "Traditional treatment of diabetes through herbs in Kutch district, Gujrat state," Planta Indica, vol. 1, no. 1, pp. 16-21, 2005.

[72] T. A. Ndarubu, O. S. Chiamaka, S. Alfa et al., "Phytochemicals, hypoglycemic and hypolipidemic effects of methanol leaf extract of Hibiscus sabdariffa in alloxan induced diabetic rats," GSC Biological and Pharmaceutical Sciences, vol. 8, no. 3, pp. 70-78, 2019.

[73] M. H. Omar, N. Shamsahal, H. Muhammad, W. A. N. Wan Ahmad, and M. I. Wasiman, "Anti-obesity and haematological effects of Malaysia Hibiscus sabdariffa L. aqueous extract on obese sprague dawley rats," Functional Foods in Health and Disease, vol. 8, no. 6, p. 340, 2018. 
[74] T.-W. Huang, C.-L. Chang, and E.-S. Kao, "Effect ofHibiscus sabdariffaextract on high fat diet-induced obesity and liver damage in hamsters," Food \& Nutrition Research, vol. 59, no. 1, Article ID 29018, 2015.

[75] D. Abdalla, I. F. Peréz-Ramírez, J. Pérez-Jiménez, and G. M. R. Nava, "Comparison of the bioactive potential of roselle (Hibiscus sabdariffa L.) calyx and its by-product: phenolic characterization by UPLC-QTOF MS and their antiobesity effect in vivo," Food Research International, vol. 126, Article ID 108589, 2019.

[76] J. W. Yun, "Possible anti-obesity therapeutics from nature - a review," Phytochemistry, vol. 71, no. 14-15, pp. 1625-1641, 2010. 